\title{
Higgs-boson masses and mixing matrices in the NMSSM: analysis of on-shell calculations
}

\author{
P. Drechsel ${ }^{1, \mathrm{a}}$, R. Gröber ${ }^{2,3, \mathrm{~b}}$, S. Heinemeyer ${ }^{4,5,6, \mathrm{c}}$, M. Mühlleitner ${ }^{7, \mathrm{~d}}$, H. Rzehak ${ }^{8, \mathrm{e}}$, G. Weiglein ${ }^{1, \mathrm{f}}$ \\ ${ }^{1}$ DESY, Notkestraße 85, 22607 Hamburg, Germany \\ 2 Department of Physics, Institute for Particle Physics Phenomenology, Durham University, Durham DH1 3LE, UK \\ ${ }^{3}$ INFN, Sezione di Roma Tre, Via della Vasca Navale 84, 00146 Rome, Italy \\ ${ }^{4}$ Campus of International Excellence UAM+CSIC, Cantoblanco, 28049 Madrid, Spain \\ ${ }^{5}$ Instituto de Física Teórica, (UAM/CSIC), Universidad Autónoma de Madrid, Cantoblanco, 28049 Madrid, Spain \\ ${ }^{6}$ Instituto de Física de Cantabria (CSIC-UC), 39005 Santander, Spain \\ ${ }^{7}$ Institute for Theoretical Physics, Karlsruhe Institute of Technology, 76128 Karlsruhe, Germany \\ ${ }^{8}$ CP3-Origins, University of Southern Denmark, Campusvej 55, 5230 Odense M, Denmark
}

Received: 23 February 2017 / Accepted: 17 May 2017 / Published online: 2 June 2017

(C) The Author(s) 2017. This article is an open access publication

\begin{abstract}
We analyze the Higgs-boson masses and mixing matrices in the NMSSM based on an on-shell (OS) renormalization of the gauge-boson and Higgs-boson masses and the parameters of the top/scalar top sector. We compare the implementation of the OS calculations in the codes NMSSMCALC and NMSSM-FeynHiggs up to $\mathcal{O}\left(\alpha_{t} \alpha_{s}\right)$. We identify the sources of discrepancies at the one- and at the twoloop level. Finally we compare the OS and $\overline{\mathrm{DR}}$ evaluation as implemented in NMSSMCALC. The results are important ingredients for an estimate of the theoretical precision of Higgs-boson mass calculations in the NMSSM.
\end{abstract}

\section{Introduction}

The experimental value of the mass of the discovered Higgs boson [1],

$m_{H}=125.09 \pm 0.21$ (stat.) \pm 0.11 (syst.) $\mathrm{GeV}$,

has an uncertainty of just a few permille so that only four years after the discovery of the Higgs boson its mass has become an electroweak precision observable. In order to make full use of this high-accuracy measurement each prediction for this quantity should be provided with a similar pre-

\footnotetext{
a e-mail: peter.drechsel@desy.de

b e-mail: ramona.groeber@durham.ac.uk

c e-mail: Sven.Heinemeyer@cern.ch

de-mail: milada.muehlleitner@kit.edu

e e-mail: rzehak@cp3.sdu.dk

f e-mail: Georg.Weiglein@ desy.de
}

cision. Furthermore, for a reliable calculation of the Higgsboson mass it is important to make a solid estimate for the theoretical uncertainty of the available prediction. Two different sources for theoretical uncertainties exist in the Higgsboson mass predictions. One is due to the experimental errors of the standard model (SM) input parameters ("parametric uncertainties"), the other are unknown higher-order corrections in the Higgs-boson mass calculation itself ("intrinsic uncertainties").

Supersymmetry (SUSY) is one of the most attractive solutions to several shortcomings of the SM. It can solve the hierarchy problem, provides a Dark Matter candidate and leads to a unification of the gauge couplings, thus paving the way to a Grand Unified Theory. The most frequently studied realizations of SUSY are the Minimal Supersymmetric Standard Model (MSSM) [2-5] and the Next-toMinimal Supersymmetric Standard Model (NMSSM) [621]. In contrast to the SM, in the MSSM two Higgs doublets are required. After electroweak symmetry breaking (EWSB), this results in five physical Higgs bosons. In the $\mathcal{C P}$-conserving case, these are two $\mathcal{C P}$-even Higgs bosons, one $\mathcal{C P}$-odd Higgs boson, and two charged Higgs bosons. The NMSSM Higgs sector is extended by an additional complex superfield leading to three $\mathcal{C} \mathcal{P}$-even, two $\mathcal{C P}$-odd and two charged Higgs bosons in the $\mathcal{C P}$-conserving case. Contrary to the SM, the masses of the Higgs bosons can be predicted in terms of the parameters of the model. While supersymmetric relations lead to an upper mass bound for the light $\mathcal{C P}$-even Higgs boson below $125 \mathrm{GeV}$ at tree level, the inclusion of higher-order corrections can shift the mass to the observed value. For the NMSSM Higgs masses the leading one-loop (s)top and (s)bottom contributions have been 
computed in [22-26]. The full one-loop corrections in the $\overline{\mathrm{DR}}$ scheme including all contributions of the NMSSM without flavour mixing have been given in [27,28], in a mixed $\overline{\mathrm{DR}} / \mathrm{OS}$ scheme in [29-31]. Two-loop corrections in the $\overline{\mathrm{DR}}$ scheme of $\mathcal{O}\left(\alpha_{s}\left(\alpha_{t}+\alpha_{b}\right)\right)$ for zero external momentum were given in [27]. Furthermore, at the two-loop level pure fermion/sfermion contributions of $\mathcal{O}\left(\left(\alpha_{t}+\alpha_{b}+\alpha_{\tau}\right)^{2}\right)$, and contributions from the Higgs/higgsino sector in the gaugeless limit of $\mathcal{O}\left(\left(\alpha_{\lambda}+\alpha_{\kappa}\right)^{2}\right)$ as well as mixed contributions from the latter two sectors of $\mathcal{O}\left(\alpha_{\lambda} \alpha_{t}, \alpha_{\lambda} \alpha_{b}\right)$ have become available in [32]. Two-loop corrections in a mixed $\overline{\mathrm{DR}} / \mathrm{OS}$ scheme of $\mathcal{O}\left(\alpha_{s}\left(\alpha_{t}+\alpha_{b}\right)\right)$ have been published in [33]. However, both in the MSSM and the NMSSM, the theoretical uncertainties of the current predictions for the mass of the SM-like Higgs boson are significantly larger than the experimental error. For the MSSM detailed estimates for theoretical uncertainties of the Higgs-mass predictions are available, see e.g. [34,35]. Automated estimates of the theoretical uncertainties depending on the considered parameter point within the MSSM can, e.g., be performed with FeynHiggs [34,36-42].

For the NMSSM several public spectrum generators are available that provide an automated calculation of the Higgs-boson masses: FlexiblesUSY [43], Flexible EFTHiggs [44], NMSSMCALC [45,46], NMSSMTOols [47-49], SOFTSUSY [50-52] and SPheno [53,54]. The results obtained by the different codes for the same set of input parameters can differ by several GeV [55].

A first step towards investigating the theoretical uncertainties for NMSSM Higgs-mass predictions focusing on calculations using a pure $\overline{\mathrm{DR}}$ renormalization has been performed in [55]. In this publication the aforementioned tools (except for FlexibleEFTHiggs, which did not exist then) were used to calculate NMSSM Higgs-boson masses for six sample scenarios with different physical properties. The sources for the differences between the codes have been identified, and after modifying the codes to use the same approximations they agree at the level of $\mathcal{O}(10 \mathrm{MeV}$ ) (for the same set of higher-order corrections). However, this technical agreement does not allow one to draw conclusions on the remaining theoretical uncertainties from unknown higher-order corrections. In particular, the comparison in [55] did not account for differences resulting from the use of different renormalization schemes. Among the tested tools only NMSSMCALC offers the option to use another renormalization scheme, namely a mixed $\overline{\mathrm{DR}} / \mathrm{on}$-shell (OS) scheme. The results in this scheme were not considered in the comparison of [55]. In the present work, we will address this issue by comparing codes incorporating a $\overline{\mathrm{DR}} / \mathrm{OS}$ scheme, namely NMSSMCALC and the NMSSM-extended version of FeynHiggs [31]. We stick here exclusively to the codes NMSSMCALC and FeynHiggs, with the latter applying a mixed $\overline{\mathrm{DR}} / \mathrm{OS}$ renormalization scheme only. Concerning the comparison between the $\overline{\mathrm{DR}} / \mathrm{OS}$ mixed scheme and the pure $\overline{\mathrm{DR}}$ scheme, we investigate the differences within NMSSMCALC for the two renormalization schemes. The analysis performed in this paper yields important ingredients for an estimate of the remaining theoretical uncertainties from unknown higherorder corrections for the Higgs-boson mass calculations in the NMSSM.

The paper is organized as follows. In Sect. 2 we introduce our notation for the relevant NMSSM parameters. In Sect. 3 we describe the codes NMSSMCALC and NMSSMFeynHiggs together with the differences between them. The analyzed numerical scenarios and their treatment is described in Sect. 4. The obtained results for the masses and mixing matrices are discussed, and their differences are analyzed in Sect. 5. The conclusions can be found in Sect. 6 . A summary of the obtained numerical results is given in Appendix.

\section{The relevant NMSSM sectors}

The superpotential of the NMSSM for the third generation fermions/sfermions reads

$$
\begin{aligned}
W= & Y_{b}\left(\hat{H}_{1} \cdot \hat{Q}_{3}\right) \hat{d}_{3}+Y_{\tau}\left(\hat{H}_{1} \cdot \hat{L}_{3}\right) \hat{e}_{3}-Y_{t}\left(\hat{H}_{2} \cdot \hat{Q}_{3}\right) \hat{u}_{3} \\
& +\lambda \hat{S}\left(\hat{H}_{2} \cdot \hat{H}_{1}\right)+\frac{1}{3} \kappa \hat{S}^{3}
\end{aligned}
$$

with the left-handed quark and lepton superfields, $\hat{Q}_{3}, \hat{L}_{3}$, and the right-handed ones, $\hat{u}_{3}, \hat{d}_{3}$, and $\hat{e}_{3}$, exemplary for all three generations, and the Higgs superfields $\hat{H}_{1}, \hat{H}_{2}$ and $\hat{S}$. The $S U(2)_{\mathrm{L}}$-invariant product is denoted by a dot. Since we will focus on the $\mathcal{C P}$-conserving NMSSM in this comparison, all the Yukawa-type couplings $Y_{t}, Y_{b}, Y_{\tau}, \lambda$ and $\kappa$ can be chosen as real parameters. The scalar components $H_{1}, H_{2}$ and $S$ of the Higgs doublet and singlet superfields can be decomposed into $\mathcal{C P}$-even and $\mathcal{C P}$-odd neutral scalars $\phi_{x}$ and $\chi_{x}(x=1,2, s)$, respectively, and charged states $\phi_{i}^{ \pm}$ $(i=1,2)$. After expansion about their vacuum expectation values (vevs) $\left\langle H_{i}\right\rangle(i=1,2)$ and $\langle S\rangle$, they read

$$
\begin{aligned}
& H_{1}=\left(\begin{array}{c}
\left\langle H_{1}\right\rangle+\frac{1}{\sqrt{2}}\left(\phi_{1} \pm i \chi_{1}\right) \\
\pm \phi_{1}^{-}
\end{array}\right), \\
& H_{2}=\left(\begin{array}{c}
\phi_{2}^{+} \\
\left\langle H_{2}\right\rangle+\frac{1}{\sqrt{2}}\left(\phi_{2}+i \chi_{2}\right)
\end{array}\right), \\
& S=\langle S\rangle+\frac{1}{\sqrt{2}}\left(\phi_{s}+i \chi_{s}\right) .
\end{aligned}
$$

The plus sign in the doublet $H_{1}$ refers to the convention used in NMSSMCALC, the minus sign to the one used in NMSSM-FeynHiggs. Due to $\mathcal{C P}$-conservation the VEVs are real. Since $\hat{S}$ transforms as a singlet, the $D$-terms remain 
identical to the ones from the MSSM. Compared to the $\mathcal{C P}$ conserving MSSM the superpotential of the $\mathcal{C P}$-conserving NMSSM contains additional dimensionless parameters $\lambda$ and $\kappa$, while the $\mu$-term is absent. This term is generated effectively via the VEV of the singlet field,

$\mu_{\mathrm{eff}}=\lambda\langle S\rangle$.

It should be noted that there are two common conventions for defining the vacuum expectation values, $\langle S\rangle=v_{s} / \sqrt{2}$ and $\left\langle H_{i}\right\rangle=v_{i} / \sqrt{2}$, or $\langle S\rangle=v_{s}$ and $\left\langle H_{i}\right\rangle=v_{i}$, respectively. Both are allowed by the SLHA conventions [56,57]. The latter convention is used by FeynHiggs, while the former is used by NMSSMCALC. As in the MSSM it is convenient to define the ratio

$\tan \beta=\frac{v_{2}}{v_{1}}$.

Soft SUSY-breaking in the NMSSM gives rise to the real (in the $\mathcal{C P}$-conserving case) trilinear soft SUSY-breaking parameters $A_{\lambda}$ and $A_{\kappa}$, as well as to the soft-SUSY breaking mass term $m_{S}^{2}$ of the scalar singlet field. Together with the soft SUSY-breaking Lagrangian of the MSSM we have

$$
\begin{aligned}
\mathcal{L}_{\text {soft }}= & \mathcal{L}_{\text {soft,MSSM }}-m_{S}^{2}|S|^{2} \\
& -\left[\lambda A_{\lambda} S\left(H_{2} \cdot H_{1}\right)+\frac{1}{3} \kappa A_{\kappa} S^{3}+\text { h.c. }\right] .
\end{aligned}
$$

The MSSM soft SUSY-breaking Lagrangian, exemplary for the third generation, reads

$$
\begin{aligned}
\mathcal{L}_{\text {soft,MSSM }}= & -m_{1}^{2} H_{1}^{\dagger} H_{1}-m_{2}^{2} H_{2}^{\dagger} H_{2}-M_{\tilde{Q}_{3}}^{2} \tilde{Q}_{3}^{\dagger} \tilde{Q}_{3} \\
& -M_{\tilde{L}_{3}}^{2} \tilde{L}_{3}^{\dagger} \tilde{L}_{3}-M_{\tilde{t}_{R}}^{2} \tilde{u}_{3}^{*} \tilde{u}_{3}-M_{\tilde{b}_{R}}^{2} \tilde{d}_{3}^{*} \tilde{d}_{3} \\
& -M_{\tilde{\tau}_{R}}^{2} \tilde{e}_{3}^{*} \tilde{e}_{3}-\left(\left[Y_{\tau} A_{\tau}\left(H_{1} \cdot \tilde{L}_{3}\right) \tilde{e}_{3}^{*}\right.\right. \\
& \left.+Y_{b} A_{b}\left(H_{1} \cdot \tilde{Q}_{3}\right) \tilde{d}_{3}^{*}-Y_{t} A_{t}\left(H_{2} \cdot \tilde{Q}_{3}\right) \tilde{u}_{3}^{*}\right] \\
& + \text { h.c. })-\frac{1}{2}\left(M_{1} \tilde{B} \tilde{B}+M_{2} \tilde{W}_{i} \tilde{W}_{i}\right. \\
& \left.+M_{3} \tilde{G} \tilde{G}+\text { h.c. }\right),
\end{aligned}
$$

where the tilde in the first three lines denotes the scalar component of the corresponding superfield, $m_{1}^{2}, m_{2}^{2}, M_{\tilde{Q}_{3}}^{2}$, $M_{\tilde{t}_{R}}^{2}, M_{\tilde{b}_{R}}^{2}, M_{\tilde{L}_{3}}^{2}$ and $M_{\tilde{\tau}_{R}}^{2}$ are the soft-SUSY breaking mass parameters for the Higgs bosons, the squarks and the sleptons, respectively, and $A_{t}, A_{b}$ and $A_{\tau}$ are the soft-SUSY breaking trilinear couplings of the squarks and sleptons. The last line summarizes the soft SUSY-breaking gaugino mass terms for the $U(1)_{Y}, S U(2)_{\mathrm{L}}$, and $S U(3)_{\mathrm{c}}$ gaugino fields $\tilde{B}$, $\tilde{W}_{i}(i=1,2,3)$ and $\tilde{G}$ with the gaugino mass parameters, $M_{1}, M_{2}$ and $M_{3}$. The Higgs potential $V_{H}$ can be written in powers of the fields,

$$
\begin{aligned}
V_{H}= & \cdots-T_{\phi_{1}} \phi_{1}-T_{\phi_{2}} \phi_{2}-T_{\phi_{S}} \phi_{s} \\
& +\frac{1}{2}\left(\phi_{1}, \phi_{2}, \phi_{s}\right) \mathbf{M}_{\phi \phi}\left(\begin{array}{c}
\phi_{1} \\
\phi_{2} \\
\phi_{s}
\end{array}\right)+\frac{1}{2}\left(\chi_{1}, \chi_{2}, \chi_{s}\right) \\
& \times \mathbf{M}_{\chi \chi}\left(\begin{array}{c}
\chi_{1} \\
\chi_{2} \\
\chi_{s}
\end{array}\right)+\left(\phi_{1}^{-}, \phi_{2}^{-}\right) \mathbf{M}_{\phi^{ \pm} \phi^{ \pm}}\left(\begin{array}{c}
\phi_{1}^{+} \\
\phi_{2}^{+}
\end{array}\right)+\cdots,
\end{aligned}
$$

where the coefficients linear and bilinear in the fields are the tadpole parameters $T_{\phi_{1}}, T_{\phi_{2}}, T_{\phi_{S}}$ and the mass matrices $\mathbf{M}_{\phi \phi}, \mathbf{M}_{\chi \chi}$ and $\mathbf{M}_{\phi^{ \pm} \phi^{ \pm}}$, respectively. The dots denote constant terms and terms trilinear and quartic in the fields.

For the $\mathcal{C P}$-conserving case the mixing into the mass and $\mathcal{C P}$ eigenstates can be described at lowest order by the following unitary transformations:

$$
\begin{aligned}
\left(\begin{array}{l}
h_{1} \\
h_{2} \\
h_{3}
\end{array}\right) & =\mathbf{U}_{e(0)}\left(\begin{array}{l}
\phi_{1} \\
\phi_{2} \\
\phi_{s}
\end{array}\right),\left(\begin{array}{l}
A_{1} \\
A_{2} \\
G^{0}
\end{array}\right)=\mathbf{U}_{o(0)}\left(\begin{array}{l}
\chi_{1} \\
\chi_{2} \\
\chi_{s}
\end{array}\right), \\
\left(\begin{array}{l}
H^{ \pm} \\
G^{ \pm}
\end{array}\right) & =\mathbf{U}_{c(0)}\left(\begin{array}{l}
\phi_{1}^{ \pm} \\
\phi_{2}^{ \pm}
\end{array}\right) .
\end{aligned}
$$

The new fields correspond to the five neutral Higgs bosons $h_{i}$ and $A_{j}$, the charged Higgs pair $H^{ \pm}$, and the Goldstone bosons $G^{0}$ and $G^{ \pm}$. The matrices $\mathbf{U}_{\{e, o, c\}(0)}$ transform the Higgs fields such that the mass matrices $\mathbf{M}_{h h}, \mathbf{M}_{A A}$, and $\mathbf{M}_{H^{ \pm} H^{\mp}}$ are diagonalized at tree level,

$$
\begin{aligned}
& \mathbf{M}_{h h}=\mathbf{U}_{e(0)} \mathbf{M}_{\phi \phi} \mathbf{U}_{e(0)}^{\dagger}, \mathbf{M}_{A A}=\mathbf{U}_{o(0)} \mathbf{M}_{\chi \chi} \mathbf{U}_{o(0)}^{\dagger}, \\
& \mathbf{M}_{H^{ \pm} H^{\mp}}=\mathbf{U}_{c(0)} \mathbf{M}_{\phi^{ \pm} \phi^{ \pm}} \mathbf{U}_{c(0)}^{\dagger} .
\end{aligned}
$$

\section{The two codes: NMSSMCALC and FeynHiggs}

In this section we will give a brief overview about the higherorder corrections to Higgs-boson masses included in FeynHiggs and NMSSMCALC, together with the different renormalization schemes employed. We will restrict ourselves here and in the following to the CP-even Higgs sector.

\subsection{Incorporation of higher-order contributions}

The masses of the $\mathcal{C P}$-even Higgs bosons are obtained from the complex poles of the full propagator matrix. The inverse propagator matrix for the three $\mathcal{C P}$-even Higgs bosons $h_{i}$ from Eq. (9) is a $3 \times 3$ matrix which reads

$\Delta^{-1}\left(k^{2}\right)=i\left[k^{2} \mathbb{1}-\mathbf{M}_{h h}+\hat{\Sigma}_{h h}\left(k^{2}\right)\right]$.

Here, $\hat{\Sigma}_{h h}$ denotes the matrix of the renormalized self-energy corrections of the $\mathrm{CP}$-even Higgs fields. The three complex 
poles of the propagator in the $\mathcal{C P}$-even Higgs sector are given by the values of the external momentum $k^{2}$ for which the determinant of the inverse propagator-matrix vanishes,

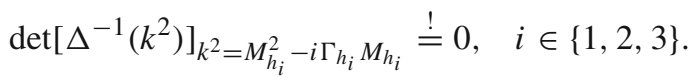

The real parts of the three poles are identified with the square of the Higgs-boson masses $M_{h_{i}}$ in the $\mathcal{C P}$-even sector, while the imaginary parts include their total widths $\Gamma_{h_{i}}$. The renormalized self-energy matrix $\hat{\Sigma}_{h h}$ at one-loop order is evaluated in NMSSMCALC [29,30] and NMSSM-FeynHiggs [31] by taking into account the full contributions from the NMSSM without flavour mixing and including momentum dependence (differences in the renormalization schemes are discussed below). At two-loop order NMSSMCALC includes the leading (S)QCD corrections from the top/stop sector of $\mathcal{O}\left(\alpha_{t} \alpha_{s}\right)$ in the NMSSM [33], while NMSSM-FeynHiggs uses all available corrections from the MSSM, which are included in the MSSM version of FeynHiggs [34,36-42], as an approximation, ${ }^{1}$

$$
\begin{aligned}
& \text { NMSSMCALC }: \hat{\boldsymbol{\Sigma}}_{h h}^{\mathrm{NC}}\left(k^{2}\right) \\
& =\left.\hat{\boldsymbol{\Sigma}}_{h h}^{(1 \mathrm{~L})}\left(k^{2}\right)\right|^{\mathrm{NMSSM}}+\left.\hat{\boldsymbol{\Sigma}}_{h h}^{(2 \mathrm{~L})}\left(k^{2}\right)\right|_{k^{2}=0} ^{\mathrm{NMSSM} \mathcal{O}\left(\alpha_{t} \alpha_{s}\right)}, \\
& \text { FeynHiggs : } \hat{\boldsymbol{\Sigma}}_{h h}^{\text {FeynHiggs }}\left(k^{2}\right) \\
& =\left.\hat{\boldsymbol{\Sigma}}_{h h}^{(1 \mathrm{~L})}\left(k^{2}\right)\right|^{\mathrm{NMSSM}}+\left[\left.\hat{\boldsymbol{\Sigma}}_{h h}^{(2 \mathrm{~L})}\left(k^{2}\right)\right|^{\mathcal{O}\left(\alpha_{t} \alpha_{s}\right)}\right. \\
& \left.\quad+\mathcal{O}\left(\alpha_{t}^{2}, \alpha_{b} \alpha_{s}, \alpha_{t} \alpha_{b}\right)+\text { resummed logs }\right]_{k^{2}=0}^{\mathrm{MSSM}} .
\end{aligned}
$$

In order to facilitate the comparison between NMSSMCALC and NMSSM-FeynHiggs at two-loop order we only include the MSSM corrections of $\mathcal{O}\left(\alpha_{t} \alpha_{s}\right)$ in NMSSM-FeynHiggs, if not stated otherwise.

The mixing-matrix elements including higher-order corrections are denoted by $U_{i j}^{h}$. Here and in the following we will suppress the loop order of the mixing matrix, but specify it in the text. They are given by the unitary matrices that diagonalize the mass-matrix at tree level, $\mathbf{U}_{e(0)}$, and the loopcorrected mass matrix for zero external momentum, $\mathbf{U}_{e(i)}$ with $i$ denoting the loop order,

$\mathbf{U}^{\mathbf{h}}=\mathbf{U}_{e(i)} \mathbf{U}_{e(0)}$,

where

$$
\begin{aligned}
& \operatorname{diag}\left(M_{h_{1}, 0}^{2}, M_{h_{2}, 0}^{2}, M_{h_{3}, 0}^{2}\right) \\
& \quad=\mathbf{U}_{e(i)}\left[\operatorname{diag}\left(m_{h_{1}}^{2}, m_{h_{2}}^{2}, m_{h_{3}}^{2}\right)+\hat{\mathbf{\Sigma}}_{h h}^{(i)}\left(k^{2}=0\right)\right] \mathbf{U}_{e(i)}^{\dagger} .
\end{aligned}
$$

\footnotetext{
${ }_{1}$ Updates beyond the FeynHiggs version 2.10.2 (used for this comparison) also take into account momentum dependent two-loop contributions $[58,59]$ and improved resummations of large logarithmic corrections [41]. These updates are not relevant for the comparison between the two codes up to $\mathcal{O}\left(\alpha_{t} \alpha_{s}\right)$.
}

Here, $m_{h_{j}}$ and $M_{h_{j}, 0}$ with $j=1,2,3$, denote the Higgsboson masses at tree-level and at higher order, respectively, i.e. including up to one-loop corrections for $i=1$ and up to two-loop contributions for $i=2$, with vanishing external momentum $k^{2}=0$. The evaluation of the mixing matrices at zero external momentum ensures the unitarity of the mixing matrices. The mixing matrices considered here differ from the wave function normalization factors for external Higgs bosons in an S-matrix element. The latter are evaluated at the complex poles of the propagators and form a non-unitary matrix. We found that the differences between the two types of matrices are small for most of the scenarios.

\subsection{Renormalization scheme: Higgs- and electroweak sectors}

The independent parameters appearing in the linear and bilinear terms of the Higgs potential in Eq. (8) have to be renormalized for the evaluation of higher-order corrections to the Higgs-boson masses. NMSSMCALC and NMSSM-FeynHiggs offer different choices for the set of independent parameters and the applied renormalization schemes [29-31,33]. For the presented work the set of common independent parameters in the Higgs sector for the comparison of the mixed $\overline{\mathrm{DR}} / \mathrm{OS}$ renormalization schemes in the two codes reads

$\underbrace{M_{Z}, M_{W}, M_{H^{ \pm}}, T_{\left.\phi_{\{1,2, s\}}\right\}}}_{\text {on-shell }}, \underbrace{\tan \beta, \lambda, \mu_{\mathrm{eff}}, \kappa, A_{k}}_{\overline{\mathrm{DR}}}$,

with the applied renormalization scheme. In NMSSMCALC the scheme can be varied: if $M_{H^{ \pm}}$is set as input parameter, it will be renormalized OS, if instead the trilinear softSUSY breaking parameter $A_{\lambda}$ is used as input parameter it is renormalized $\overline{\mathrm{DR}}$; see Sect. 5.6. The gauge-boson masses, however, are still renormalized OS, and the tadpole coefficients are renormalized such that the renormalized tadpoles vanish.

Using the former option, $M_{H^{ \pm}}$as input, up to the oneloop level the two codes differ only by their treatment of the renormalization of the coupling constant $\alpha$ in the electromagnetic sector. While for NMSSMCALC $\alpha$ is renormalized to $\alpha\left(M_{Z}\right)$, NMSSM-FeynHiggs employs a dependent renormalization scheme (employing a $\overline{\mathrm{DR}}$ renormalization of $v$ ) with a subsequent reparametrization [31] to the value $\alpha_{G_{F}}$, derived from the Fermi constant $G_{F}$ (to match exactly the FeynHiggs MSSM evaluation in the MSSM limit). This difference in the treatment of the charge renormalization at the one-loop level is formally an effect of electroweak two-loop order. In the Higgs-boson mass calculation in the MSSM limit the charge renormalization constant drops out at the discussed levels of the calculation 
Table 1 Calculational differences between the original versions of NMSSMCALC and NMSSM-FeynHiggs as used for this comparison. The values applied for the electromagnetic and strong coupling constants are stated, where $\alpha_{G_{F}}$ denotes the electromagnetic coupling constant calculated from the Fermi constant

\begin{tabular}{llll}
\hline & NMSSMCALC & & NMSSM-FeynHiggs \\
\hline 1-loop & $\alpha\left(M_{Z}\right)$ renormalized & $\leftrightarrow$ & $\alpha_{G_{F}}$ reparametrized \\
2-loop & $\alpha_{s}^{\overline{\mathrm{DR}}}\left(Q_{\text {input }}\right)$ & $\leftrightarrow$ & $\alpha_{s}^{\overline{\mathrm{MS}}}\left(m_{t}\right)$ \\
& $\mathrm{NMSSM} \mathcal{O}\left(\alpha_{t} \alpha_{s}\right)$ & $\leftrightarrow$ & $\operatorname{MSSM} \mathcal{O}\left(\alpha_{t} \alpha_{s}, \alpha_{t}^{2}, \alpha_{b} \alpha_{s}, \alpha_{t} \alpha_{b}\right)$ \\
& & + resummed logarithms \\
& &
\end{tabular}

and thus its impact is a genuine NMSSM effect. The differences between NMSSMCALC and NMSSM-FeynHiggs in the contributions to the Higgs-boson self-energies at the one-loop and the two-loop level (see below) are summarized in Table 1.

\subsection{Renormalization scheme: top/stop sector}

For the two-loop $\mathcal{O}\left(\alpha_{t} \alpha_{s}\right)$ corrections the top quark mass and the stop parameters need to be renormalized. In NMSSMCALC either the OS or the $\overline{\mathrm{DR}}$ renormalization scheme for the top/stop sector can be used. Apart from Sect. 5.6, where we indicate explicitly the renormalization scheme of the top/stop sector, we employ the OS scheme in NMSSMCALC throughout this work. In NMSSM-FeynHiggs the OS scheme is used throughout for the parameters in the stop sector. For the top-quark mass, either the OS or the $\overline{\mathrm{DR}}$ renormalization scheme can be chosen in NMSSM-FeynHiggs, and a further option is to use a reparametrization of the OS result in terms of the $\overline{\mathrm{MS}}$ mass of SM QCD.

In both programs, the OS scheme is defined by applying on-shell conditions for the respective masses, i.e. the top-quark mass $m_{t}$ and the top-squark masses $m_{\tilde{t}_{1}}$ and $m_{\tilde{t}_{2}}$. A fourth renormalization condition fixes the mixing of the squarks and can be identified with a condition for the stop mixing angle. The resulting counterterms have the same form as in the MSSM, and details can be found in Refs. [60,61]. No additional counterterms of the sbottom sector are needed since the bottom mass is set to zero in the charged Higgs self-energies.

NMSSMCALC uses the soft-SUSY breaking masses of left-handed and right-handed fields, $M_{\tilde{Q}_{3}}$ and $M_{\tilde{t}_{R}}$, as well as the trilinear coupling $A_{t}$ and calculates counterterms for these parameters corresponding to the above mentioned renormalization conditions. In this way, switching from OS to $\overline{\mathrm{DR}}$ parameters can easily be done; see Ref. [33]. NMSSM-FeynHiggs uses the same numerical input values of the soft-SUSY breaking parameters and the same OS conditions. Counterterms are employed in the stop sector for the stop masses and the stop mixing angle.

\subsection{Treatment of QCD corrections}

In NMSSMCALC the $\overline{\mathrm{DR}}$-value of the strong coupling constant $\alpha_{s}^{\overline{\mathrm{DR}}}$ is calculated at the input scale $Q$ of the parameters specified in the SLHA input file by applying the formulas for the SM given in $[62,63]$. In NMSSM-FeynHiggs the $\overline{\mathrm{MS}}$-value of the strong coupling constant $\alpha_{s}^{\overline{\mathrm{MS}}}$ is calculated at the scale $m_{t}$. In both codes the obtained value is subsequently used for the evaluation of the two-loop contributions to the Higgs boson masses. As stressed above, NMSSMCALC includes corrections of up to $\mathcal{O}\left(\alpha_{t} \alpha_{s}\right)$, and consequently for our comparison we restrict the NMSSM-FeynHiggs evaluation to this order as well. The treatment of the two-loop contributions is summarized in the lower row of Table 1.

\section{Description of the scenarios}

\subsection{The five test-point scenarios}

In Ref. [55], six test-point (TP) scenarios were proposed for the comparison of the Higgs-mass predictions obtained by different tools using the $\overline{\mathrm{DR}}$ scheme. They will also be employed here for the comparison between NMSSMCALC and NMSSM-FeynHiggs to facilitate a later comparison with Ref. [55]. The definitions of the TP scenarios are recapitulated in Table 2, where all parameters are given at the indicated scale, both at the high-scale $M_{S}$ at which they were originally defined, and at the scale of the top-quark on-shell mass $m_{t}{ }^{2}$ For completeness we repeat the different physical features of these scenarios as given in Ref. [55]:

TP1: MSSM-like point.

TP2: MSSM-like point with large stop splitting.

TP3: Point with light singlet and $\lambda$ close to the perturbativity limit.

TP4: Point with heavy singlet and $\lambda$ close to the perturbativity limit.

TP5: Point with slightly lighter singlet. Additional matter needed for perturbativity; inspired by [64].

The scenario TP6 of Ref. [55] is characterized by a very large value of $\lambda$. It will be omitted from this comparison, since the corrections beyond the $\mathcal{O}\left(\alpha_{t} \alpha_{s}\right)$ approximation can be sizeable in this case $[32,55]$. Furthermore, this scenario requires new physics well below the GUT scale to avoid

\footnotetext{
${ }^{2}$ In the original definition of the scenarios in Ref. [55] $\tan \beta$ is given by its $\overline{\mathrm{DR}}$-value at the scale of the $Z$-boson mass $M_{Z}$, while in Table 2 we give the corresponding $\overline{\mathrm{DR}}$-value at the indicated scale, either $M_{S}$ or $m_{t}$. The values for $\tan \beta$ given here are obtained with FlexibleSUSY as described in the text.
} 
Table 2 Definition of the TP scenarios. All parameters are given as $\overline{\mathrm{DR}}$ parameters at the indicated scale $Q$. All dimensionful parameters are given in $\mathrm{GeV}$. The remaining parameters, common to all points, are the soft SUSY-breaking parameters in the sfermion mass matrices, $M_{\tilde{L}_{i}}=M_{\tilde{Q}_{j}}=M_{\tilde{f}_{R}}=M_{\tilde{b}_{R}}=1500 \mathrm{GeV}$, where $i=1,2,3$, $j=1,2, f=e, \mu, \tau, d, s, u, c$, and the trilinear sfermion-Higgs coupling $A_{f}=0 \mathrm{GeV}$ at the high scale, $M_{S}=\frac{1}{2}\left(M_{\tilde{Q}_{3}}+M_{\tilde{t}_{R}}\right)$. At the scale $Q=m_{t}=172.9 \mathrm{GeV}$ we use the corresponding SUSY-breaking parameters but evolved to the scale $Q=m_{t}$. The scenario TP2 yields tachyonic stop masses at the scale $m_{t}$

\begin{tabular}{|c|c|c|c|c|c|c|c|c|c|c|c|c|c|c|}
\hline & $Q$ & $\tan \beta$ & $\lambda$ & $\kappa$ & $A_{\lambda}$ & $A_{k}$ & $\mu_{\mathrm{eff}}$ & $M_{1}$ & $M_{2}$ & $M_{3}$ & $A_{t}$ & $A_{b}$ & $M_{\tilde{Q}_{3}}$ & $M_{\tilde{t}_{R}}$ \\
\hline \multirow[t]{2}{*}{ TP1 } & $M_{S}$ & 9.599 & 0.100 & 0.100 & -10.00 & -10.00 & 900.0 & 500.0 & 1000 & 3000 & 3000 & 0 & 1500 & 1500 \\
\hline & $m_{t}$ & 9.903 & 0.098 & 0.100 & -198.6 & -9.738 & 886.6 & 478.1 & 979.4 & 3261 & 2154 & -585.2 & 1907 & 1838 \\
\hline \multirow[t]{2}{*}{ TP2 } & $M_{S}$ & 9.621 & 0.050 & 0.100 & -200.0 & -200.0 & 1500 & 1000 & 2000 & 2500 & -2900 & 0 & 2500 & 500 \\
\hline & $m_{t}$ & - & - & - & - & - & - & - & - & - & - & - & - & - \\
\hline \multirow[t]{2}{*}{ TP3 } & $M_{S}$ & 2.881 & 0.670 & 0.100 & 650.0 & -10.00 & 200.0 & 200.0 & 400.0 & 2000 & 1000 & 1000 & 1000 & 1000 \\
\hline & $m_{t}$ & 2.967 & 0.648 & 0.097 & 574.0 & -43.68 & 195.3 & 192.4 & 391.9 & 2145 & 619.8 & 691.5 & 1233 & 1211 \\
\hline \multirow[t]{2}{*}{ TP4 } & $M_{S}$ & 1.920 & 0.670 & 0.200 & 405.0 & 0 & 200.0 & 120.0 & 200.0 & 1500 & 1000 & 1000 & 750.0 & 750.0 \\
\hline & $m_{t}$ & 1.975 & 0.649 & 0.195 & 344.3 & 0.195 & 195.3 & 116.0 & 195.7 & 1591 & 726.5 & 801.5 & 891.9 & 874.4 \\
\hline \multirow[t]{2}{*}{ TP5 } & $M_{S}$ & 2.864 & 0.670 & 0.200 & 570.0 & -25.00 & 200.0 & 135.0 & 200.0 & 1400 & 0 & 0 & 1500 & 1500 \\
\hline & $m_{t}$ & 2.967 & 0.643 & 0.193 & 549.3 & -63.47 & 194.4 & 128.7 & 194.3 & 1526 & -234.8 & -251.9 & 1579 & 1546 \\
\hline
\end{tabular}

the non-perturbative regime. All TP scenarios, using a $\overline{\mathrm{DR}}$ renormalization, contain a SM-like Higgs field with a mass predicted at the two-loop level of around $125 \mathrm{GeV}$.

The measured value of the discovered Higgs boson of $\sim 125 \mathrm{GeV}$ is at the weak scale. The diagrammatic corrections to the Higgs-boson self-energies in NMSSMCALC and NMSSM-FeynHiggs are such that the full particle spectrum of the model is incorporated in the loop contributions. This approach is motivated by scenarios where the SUSY scale is relatively low, i.e. not widely separated from the weak scale, and where there is no large hierarchy among the SUSY particle masses. Within this context, the diagrammatic approach yields general results for arbitrary values of the involved parameters. In contrast, effective field theory (EFT) methods are designed for the treatment of largescale splittings within the calculation. ${ }^{3}$ Within the context of the diagrammatic calculations, we investigate two possible treatments of the scale of the SUSY parameters. We will perform the conversion of the $\overline{\mathrm{DR}}$ to $\mathrm{OS}$ parameters and (for the calculation in the $\overline{\mathrm{DR}}$ scheme) the evaluation of the Higgs-boson masses for the given scenarios both at their original scale $M_{S}=\frac{1}{2}\left(M_{\tilde{O}_{3}}+M_{\tilde{t}_{R}}\right)$, the arithmetic mean of the two diagonal soft SUSY-breaking mass parameters in the scalar top mass matrix, as well as at the scale of the OS top-quark mass $m_{t}=172.9 \mathrm{GeV}$. In the latter case this means in particular that the parameters are first evolved from their original scale $M_{S}$ to the scale $m_{t}$ with FlexibleSUSY, with renormalization group equations generated by SARAH [65-67], before they are converted to OS parameters.

\footnotetext{
${ }^{3}$ As stated above, the contributions from resummed logarithms in FeynHiggs obtained using EFT methods are not included in our comparison.
}

The parameters at the scale $m_{t}$ are given in Table 2, together with the original parameters at the scale $M_{S}$. The scenario TP2 yields tachyonic stop masses at the scale $m_{t}$. Consequently, TP2 is evaluated only at the scale $M_{S}$. Note that even though we perform an OS renormalization of the parameters of the top/stop sector, we do not obtain identical OS stop masses at both scales for the same scenario. For the OS scheme it would be preferable to define the OS stop masses as input parameters, rather than the $\overline{\mathrm{DR}}$ soft-SUSY breaking parameters. Our goal is, however, to ensure compatibility with Ref. [55], hence we use the $\overline{\mathrm{DR}}$ input parameters and convert them to OS parameters at the specific scales.

\subsection{Conversion from $\overline{\mathbf{D R}}$ to OS parameters}

In NMSSMCALC it is possible to perform calculations with either the OS or the $\overline{\mathrm{DR}}$ renormalization scheme in the top/stop sector. In NMSSM-FeynHiggs the OS scheme is mandatory for the scalar top quarks, while for the top-quark mass the OS scheme, the $\overline{\mathrm{DR}}$ scheme and a reparametrization of the OS result in terms of the $\overline{\mathrm{MS}}$ mass of SM QCD can be chosen. Both codes are capable of converting $\overline{\mathrm{DR}}$ input parameters at their given scale into OS parameters by using the well-known OS shifts of the MSSM [61,68]. ${ }^{4}$ For our comparison we used the routines of only one code, NMSSMCALC, in order to prevent effects that originate from different implementations of these shifts. The shifts in NMSSMCALC are computed as

$X^{(\mathrm{OS})}=X^{(\overline{\mathrm{DR}})}-\delta X^{\mathrm{fin}} \quad$ with $\quad X=M_{\tilde{Q}_{3}}, M_{\tilde{t}_{R}}, A_{t}$,

\footnotetext{
$\overline{4}$ Since only corrections of up to $\mathcal{O}\left(\alpha_{t} \alpha_{s}\right)$ are discussed in this work, only one-loop shifts of $\mathcal{O}\left(\alpha_{s}\right)$ for the scalar top sector are necessary. These are identical in the MSSM and the NMSSM.
} 
Table 3 OS parameters in the TP scenarios obtained by the routines of NMSSMCALC. Here $A_{t}^{\mathrm{OS}}$ is the trilinear soft-breaking parameter in the stop sector, while $M_{\tilde{Q}_{3}}^{\mathrm{OS}}$ and $M_{\tilde{t}_{R}}^{\mathrm{OS}}$ are the soft SUSY-breaking mass parameters in the stop sector for the $S U(2)_{\mathrm{L}}$ doublet and singlet, respec- tively. $M_{H^{ \pm}}$denotes the OS renormalized mass of the charged Higgs boson, and $m_{\tilde{t}_{i}}^{\mathrm{OS}}$ denotes the obtained OS masses of the two top squarks. All parameter values are given in $\mathrm{GeV}$

\begin{tabular}{|c|c|c|c|c|c|c|c|c|c|c|}
\hline \multirow[t]{2}{*}{$Q$} & \multicolumn{2}{|l|}{ TP1 } & \multicolumn{2}{|l|}{$\underline{\mathrm{TP} 2}$} & \multicolumn{2}{|l|}{ TP3 } & \multicolumn{2}{|l|}{$\underline{\text { TP4 }}$} & \multicolumn{2}{|l|}{ TP5 } \\
\hline & $M_{S}$ & $m_{t}$ & $M_{S}$ & $m_{t}$ & $M_{S}$ & $m_{t}$ & $M_{S}$ & $m_{t}$ & $M_{S}$ & $m_{t}$ \\
\hline$A_{t}^{\mathrm{OS}}$ & 2758 & 2651 & -2525 & - & 940.6 & 933.4 & 953.0 & 917.2 & -87.80 & -25.83 \\
\hline$M_{\tilde{Q}_{3}}^{\mathrm{OS}}$ & 1507 & 1181 & 2542 & - & 1003 & 856.2 & 750.4 & 655.5 & 1547 & 1494 \\
\hline$M_{\tilde{t}_{\mathrm{R}}}^{\mathrm{OS}}$ & 1507 & 1055 & 513 & - & 1003 & 822.2 & 750.4 & 629.5 & 1547 & 1460 \\
\hline$M_{H^{ \pm}}$ & 2759 & 2755 & 6373 & - & 641.6 & 642.1 & 455.7 & 455.4 & 615.4 & 617.3 \\
\hline$m_{\tilde{t}_{1}}^{\mathrm{OS}}$ & 1355 & 904.3 & 507.0 & - & 939.9 & 761.7 & 667.2 & 547.0 & 1548 & 1469 \\
\hline$m_{\tilde{t}_{2}}^{\mathrm{OS}}$ & 1661 & 1321 & 2554 & - & 1088 & 940.9 & 859.4 & 764.2 & 1565 & 1504 \\
\hline
\end{tabular}

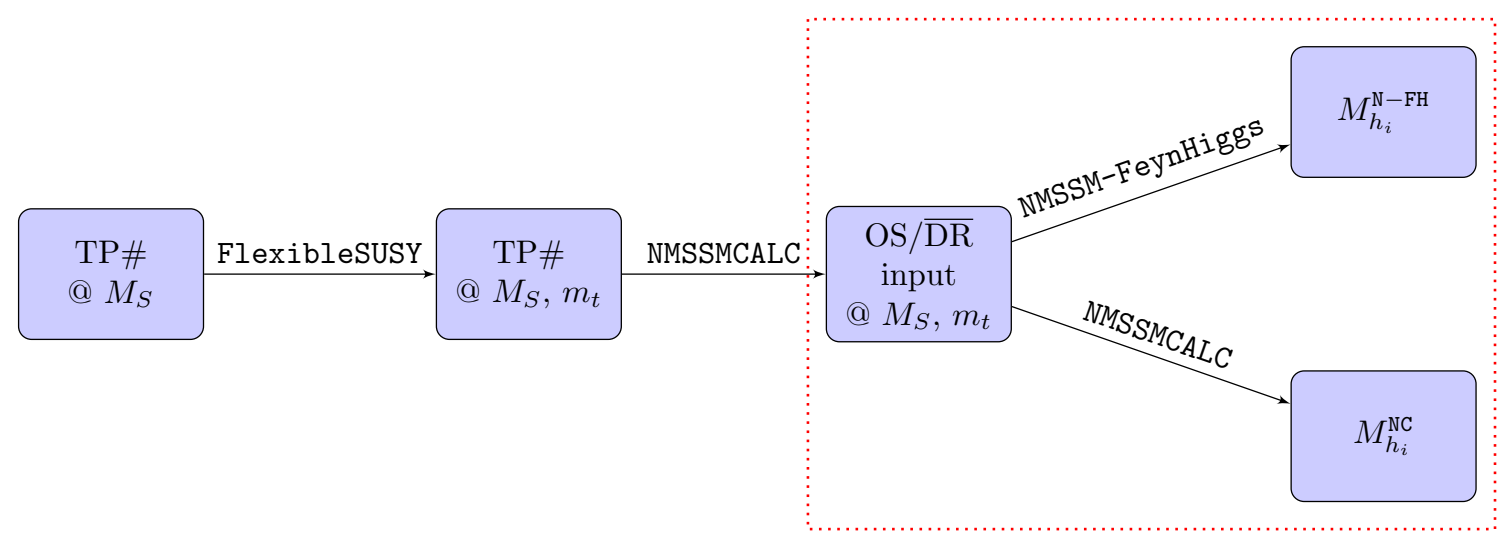

Fig. 1 Steps performed to obtain predictions for the masses and mixing matrices of the $\mathcal{C P}$-even Higgs fields at the scale $Q$. The red dashed line denotes the actual calculation of the Higgs masses. It is independent of the RGE evolution and the OS conversion of the input parameters

where $X^{(\overline{\mathrm{DR}})}$ and $\delta X^{\text {fin }}$ both depend on the $\overline{\mathrm{DR}}$ scale. The shift $\delta X^{\text {fin }}$ denotes the finite part of the respective counterterm obtained in the OS scheme as given in Ref. [33]. They are computed iteratively by inserting the obtained OS parameters, until convergence is reached.

The current version of NMSSM-FeynHiggs requires an OS mass for the charged Higgs boson as input. In order to obtain this quantity from the $\overline{\mathrm{DR}}$ parameters specified in Table 2, we used routines implemented in NMSSMCALC. Those routines calculate a two-loop pole mass for the charged Higgs boson from the given input parameters. We use the $\overline{\mathrm{DR}}$ option for the renormalization of the top/stop sector for this computation. The result is treated as the OS mass for this comparison and is used as an input value for both codes, NMSSM-FeynHiggs and NMSSMCALC. ${ }^{5}$ The OS shifted stop-sector parameters and the resulting stop masses as obtained with the routines of NMSSMCALC are given in Table 3. An overview of the procedure of how the

\footnotetext{
5 The choice of using $\overline{\mathrm{DR}}$ stop-sector parameters in the evaluation of $M_{H^{ \pm}}$(and not the ones converted to OS) later facilitates the comparison with a pure $\overline{\mathrm{DR}}$ calculation.
}

Higgs-boson masses and mixing matrices are obtained from the original definition of the scenarios TP1-TP5 is given in Fig. 1. The transition between $\overline{\mathrm{DR}}$ and OS parameters can give rise to significant shifts in the case some of the involved SUSY masses are heavy; see e.g. the discussion in Refs. [68,69].

\section{Predictions for masses and mixing matrices of the $\mathcal{C P}$-even scalars}

In this section we analyze the differences in the predictions for the $\mathcal{C} \mathcal{P}$-even Higgs boson masses and mixing matrices from NMSSM-FeynHiggs and NMSSMCALC. We start by a comparison of the "out-of-the-box" results including the corrections of up to $\mathcal{O}\left(\alpha_{t} \alpha_{s}\right)$, where sizeable differences show up. In order to understand the origin of these differences we then perform a comparison at the oneloop level, where we find that part of the differences can be attributed to the different renormalization of the electroweak sector in the two codes. This difference, which is of the order of unknown electroweak two-loop corrections, 
Table 4 Mass predictions for the $\mathcal{C P}$-even scalars for TP1-TP5 when using the indicated versions of NMSSMCALC and NMSSM-FeynHiggs as specified in Sect. 5.1. The mass values of the SM-like scalar are written in bold fonts, those of the singlet-like scalar in italics

\begin{tabular}{|c|c|c|c|c|c|c|c|c|c|c|c|}
\hline & \multirow[t]{2}{*}{$Q$} & \multicolumn{2}{|l|}{ TP1 } & \multicolumn{2}{|l|}{ TP2 } & \multicolumn{2}{|l|}{ TP3 } & \multicolumn{2}{|l|}{ TP4 } & \multicolumn{2}{|l|}{ TP5 } \\
\hline & & $M_{S}$ & $m_{t}$ & $M_{S}$ & $m_{t}$ & $M_{S}$ & $m_{t}$ & $M_{S}$ & $m_{t}$ & $M_{S}$ & $m_{t}$ \\
\hline \multirow[t]{2}{*}{$h_{1}$} & NC OS & 121.84 & 113.47 & 120.42 & - & 89.92 & 88.81 & 126.44 & 126.65 & 119.54 & 117.63 \\
\hline & $\mathrm{N}-\mathrm{FH}$ & 115.70 & 113.20 & 114.12 & - & 89.67 & 89.36 & 126.17 & 126.29 & 118.47 & 117.95 \\
\hline \multirow[t]{2}{*}{$h_{2}$} & NC OS & 1797.45 & 1797.57 & 5951.36 & - & 126.16 & 125.80 & 143.32 & 142.73 & 124.44 & 123.51 \\
\hline & $\mathrm{N}-\mathrm{FH}$ & 1797.45 & 1797.62 & 5951.36 & - & 124.55 & 125.02 & 143.11 & 142.68 & 122.93 & 123.10 \\
\hline \multirow[t]{2}{*}{$h_{3}$} & NC OS & 2755.73 & 2752.14 & 6370.77 & - & 652.60 & 652.70 & 467.89 & 467.35 & 627.18 & 628.72 \\
\hline & $\mathrm{N}-\mathrm{FH}$ & 2755.79 & 2752.25 & 6370.85 & - & 652.17 & 652.65 & 467.10 & 467.33 & 626.59 & 628.76 \\
\hline
\end{tabular}

can be adjusted by an appropriate reparametrization of the NMSSM-FeynHiggs result. We then continue with an analysis at the two-loop level, where we investigate the effect of the strong coupling constant and the genuine NMSSM corrections to the Higgs boson self-energies. Finally we compare the results obtained with the OS version of NMSSMCALC with the $\overline{\mathrm{DR}}$ calculation. The identification of the various sources of differences between the different calculations are important ingredients for a reliable estimate of the intrinsic uncertainties in the Higgs-boson mass and mixing-matrix calculations in the NMSSM. A summary of the obtained numerical results of this section is given in Appendix.

\section{1 "Out-of-the-box" results}

In the first step of our comparison the masses and mixing matrices of the $\mathcal{C P}$-even Higgs sector are evaluated for the TP scenarios with the "out-of-the-box" versions of NMSSMCALC and NMSSM-FeynHiggs, restricting the two-loop corrections in the latter code to $\mathcal{O}\left(\alpha_{t} \alpha_{s}\right)$ (cf. Sect. 3.1). For the results of NMSSMCALC we used the "out-of-the-box" version with the on-shell renormalization scheme for the top/stop sector and the charged Higgs mass (see Sect. 4.2), labeled "NC OS". The OS parameters used as numerical input for both codes have been specified in Table 3 .

The obtained numerical results for the masses are given in Table 4, the results for the mixing-matrix elements, see Eq. (14), are given in Table 5. For all TP scenarios except for TP5 we identify the field $h_{i}$ with the largest value for $\left|U_{i 2}^{h}\right|$ as the SM-like field, since it has the largest coupling to the top quark. We refer to the field $h_{i}$ with the largest value for $\left|U_{i 3}^{h}\right|$ as the singlet-like field. For TP5 both lighter fields have similar or sizeable values for $\left|U_{i 2}^{h}\right|$ and $\left|U_{i 3}^{h}\right|$, in particular at the scale $m_{t}$. In this case we refer to the field $h_{2}$ with the mass closer to $125 \mathrm{GeV}$ as SM-like and to the lighter field $h_{1}$ as singlet-like (for the two-loop results considered here).

In Fig. 2 the difference $\Delta m=M_{h}^{\mathrm{NC}}-M_{h}^{\mathrm{N}-\mathrm{FH}}$ between the mass predictions obtained with the codes "NC OS" and "N-FH" are shown. All values are given for two input scales
$Q$, the SUSY mass scale $Q=M_{S}$ in lighter (green) bars and the top-quark mass scale $Q=m_{t}=172.9 \mathrm{GeV}$ in darker (blue) bars.

When comparing the Higgs masses obtained with NMSSM-FeynHiggs and NMSSMCALC, we find that for the SM-like field the difference between the mass predictions of the two codes can be larger than $6 \mathrm{GeV}$ for the scenarios TP1 and TP2 and the evaluation at the high-scale $M_{S}$. These differences between the mass predictions obtained at the two-loop level appear to be unusually large. The origin of these differences will be addressed in the following sections. For the evaluations at the low-scale $m_{t}$, however, the differences between the codes do not exceed $1 \mathrm{GeV}$ for all scenarios (as mentioned above, the scenario TP2 is not evaluated at the scale $m_{t}$ in our numerical analysis, as it yields tachyonic stop masses). The difference between the masses of the heaviest fields, which are always doublet-like in all TP scenarios, remain rather small with less than $0.8 \mathrm{GeV}$, which is at the permille level for the considered scenarios. For scenarios with a singlet-like field that is heavier than the SM-like field, i.e. TP1, TP2 and TP4, the absolute difference between the mass predictions for the singlet-like field remains below $0.25 \mathrm{GeV}$, while for a lighter singlet-field the differences can be as large as $1.1 \mathrm{GeV}$. The mixing-matrix elements of the SM-like Higgs boson obtained by the two codes agree within $\approx 2 \%$ for the scenarios TP1-TP3. For the singlet-like Higgs boson the differences in the mixing-matrix elements, on the other hand, can be substantial in these scenarios. In case of the heavy Higgs $h_{3}$ the matrix elements differ by at most 10\%. For TP4 and TP5 we find good agreement for most entries, but larger differences, up to a factor of 4 , can occur for matrix elements that are themselves small. In general, we find that the differences for the mixing-matrix elements seem to be by far larger than for the mass predictions, where the relative differences never exceed $6 \%$. This is in particular the case for TP4 and TP5, where we have large singlet admixtures to the SM-like Higgs field with a mass around $125 \mathrm{GeV}$. In these scenarios the Higgs masses and mixing matrices appear to be very sensitive to relatively small changes of the parameters. We remark that this can 
Table 5 Absolute values for the mixing-matrix elements of the $\mathcal{C P}$-even scalar sector for TP1-TP5 when using the indicated versions of NMSSMCALC and NMSSM-FeynHiggs as specified in the text; see Sect. 5.1

\begin{tabular}{|c|c|c|c|c|c|c|c|c|c|c|c|}
\hline \multirow[t]{2}{*}{$i$} & \multirow[t]{2}{*}{$Q$} & & \multicolumn{3}{|l|}{ TP1 } & \multicolumn{3}{|l|}{ TP2 } & \multicolumn{3}{|l|}{ TP3 } \\
\hline & & & $\left|U_{i 1}^{h}\right|$ & $\left|U_{i 2}^{h}\right|$ & \multirow{2}{*}{$\frac{\left|U_{i 3}^{h}\right|}{0.0076}$} & \multirow{2}{*}{$\frac{\left|U_{i 1}^{h}\right|}{0.1034}$} & \multirow{2}{*}{$\frac{\left|U_{i 2}^{h}\right|}{0.9946}$} & $\left|U_{i 3}^{h}\right|$ & $\left|U_{i 1}^{h}\right|$ & \multirow{2}{*}{$\frac{\left|U_{i 2}^{h}\right|}{0.1994}$} & \multirow{2}{*}{$\frac{\left|U_{i 3}^{h}\right|}{0.9549}$} \\
\hline \multirow[t]{4}{*}{1} & \multirow[t]{2}{*}{$M_{S}$} & NC OS & 0.1039 & 0.9946 & & & & 0.0004 & 0.2199 & & \\
\hline & & $\mathrm{N}-\mathrm{FH}$ & 0.1039 & 0.9946 & 0.0071 & 0.1034 & 0.9946 & 0.0004 & 0.2134 & 0.2064 & 0.9549 \\
\hline & \multirow[t]{2}{*}{$m_{t}$} & NC OS & 0.1006 & 0.9949 & 0.0073 & - & - & - & 0.2236 & 0.2210 & 0.9493 \\
\hline & & $\mathrm{N}-\mathrm{FH}$ & 0.1006 & 0.9949 & 0.0071 & - & - & - & 0.2245 & 0.2264 & 0.9478 \\
\hline 2 & $M_{S}$ & NC OS & 0.0075 & 0.0068 & 0.9999 & 0.0096 & 0.0006 & 1.0000 & 0.2797 & 0.9249 & 0.2575 \\
\hline & & $\mathrm{N}-\mathrm{FH}$ & 0.0071 & 0.0064 & 1.0000 & 0.0090 & 0.0005 & 1.0000 & 0.2820 & 0.9228 & 0.2625 \\
\hline & $m_{t}$ & NC OS & 0.0075 & 0.0066 & 0.9999 & - & - & - & 0.2659 & 0.9232 & 0.2775 \\
\hline & & $\mathrm{N}-\mathrm{FH}$ & 0.0072 & 0.0064 & 1.0000 & - & - & - & 0.2656 & 0.9216 & 0.2831 \\
\hline 3 & $M_{S}$ & NC OS & 0.9946 & 0.1040 & 0.0068 & 0.9946 & 0.1034 & 0.0096 & 0.9346 & 0.3237 & 0.1476 \\
\hline & & $\mathrm{N}-\mathrm{FH}$ & 0.9946 & 0.1039 & 0.0064 & 0.9946 & 0.1034 & 0.009 & 0.9354 & 0.3253 & 0.1388 \\
\hline & $m_{t}$ & NC OS & 0.9949 & 0.1006 & 0.0068 & - & - & - & 0.9377 & 0.3144 & 0.1476 \\
\hline & & $\mathrm{N}-\mathrm{FH}$ & 0.9949 & 0.1006 & 0.0065 & - & - & - & 0.9376 & 0.3153 & 0.1467 \\
\hline$i$ & $Q$ & & $\underline{\mathrm{T}}$ & & & & TP5 & & & & \\
\hline & & & & & $\left|U_{i 2}^{h}\right|$ & $\left|U_{i 3}^{h}\right|$ & $\left|U_{i 1}^{h}\right|$ & $\left|U_{i 2}^{h}\right|$ & & & \\
\hline 1 & $M_{S}$ & $\mathrm{NC}$ & & 813 & 0.7432 & 0.4648 & 0.2845 & 0.3943 & 0.87 & & \\
\hline & & $\mathrm{N}-\mathrm{F}$ & & 873 & 0.8022 & 0.3448 & 0.3540 & 0.7764 & 0.52 & & \\
\hline & $m_{t}$ & $\mathrm{NC}$ & & 766 & 0.7886 & 0.3885 & 0.3393 & 0.6991 & 0.62 & & \\
\hline & & $\mathrm{N}-\mathrm{F}$ & & 788 & 0.8104 & 0.3377 & 0.3390 & 0.7204 & 0.60 & 51 & \\
\hline 2 & $M_{S}$ & $\mathrm{NC}$ & & 895 & 0.4858 & 0.8694 & 0.2224 & 0.8594 & 0.46 & 03 & \\
\hline & & $\mathrm{N}-\mathrm{F}$ & & 334 & 0.3775 & 0.9254 & 0.0564 & 0.5387 & 0.8 & 106 & \\
\hline & $m_{t}$ & $\mathrm{NC}$ & & 411 & 0.4215 & 0.9059 & 0.0882 & 0.6425 & 0.76 & 612 & \\
\hline & & $\mathrm{N}-\mathrm{F}$ & & 171 & 0.3761 & 0.9264 & 0.0819 & 0.6181 & 0.78 & & \\
\hline 3 & $M_{S}$ & $\mathrm{NC}$ & & 720 & 0.4600 & 0.1673 & 0.9325 & 0.3253 & 0.15 & & \\
\hline & & $\mathrm{N}-\mathrm{F}$ & & 726 & 0.4625 & 0.1571 & 0.9335 & 0.3270 & 0.12 & & \\
\hline & $m_{t}$ & $\mathrm{NC}$ & & 782 & 0.4477 & 0.1685 & 0.9365 & 0.3137 & 0.15 & & \\
\hline & & $\mathrm{N}-\mathrm{F}$ & & 778 & 0.4493 & 0.1662 & 0.9372 & 0.3146 & 0.15 & & \\
\hline
\end{tabular}

lead to different conclusions whether a parameter point is excluded or not by LHC data.

In the following we will analyze the observed differences in more detail. We will start with a discussion of the chosen test-point TP1 and a study of the differences at the one-loop level.

\subsection{The test-point TP1}

In Table 4 very large differences between the mass prediction in the MSSM-like scenario TP1 can be found when comparing the result for the SM-like $\mathcal{C} \mathcal{P}$-even Higgs field obtained with the same code at either the scale $M_{S}$ or $m_{t}$. They can be as large as $\approx 8.5 \mathrm{GeV}$ for the SM-like scalar with NMSSMCALC and $\approx 3 \mathrm{GeV}$ for the heavy scalar with both codes (it should be noted, of course, that for the heavy scalar this amounts to a much smaller relative effect than for the SMlike scalar). These different results at two different scales may seem surprising since the physical situation before and after the evolution of the parameters of the scenario should be identical. Furthermore, we observed for TP1 that changing the renormalization scheme from an OS renormalization to a $\overline{\mathrm{DR}}$ renormalization in the top/stop sector for TP1 at $Q=m_{t}$, as in Sect. 5.6, changes the mass of the lightest Higgs boson by $8.7 \mathrm{GeV} .^{6}$ It can be seen from Table 3 that the running from $M_{S}$ to $m_{t}$, which we have performed with FlexibleSUSY, and the conversion from the $\overline{\mathrm{DR}}$ to the OS scheme, which we have carried out as described in Sect. 4.2, gives rise to large shifts of the OS parameters of the scalar top sector in this scenario. These large effects are induced by the large splitting between the gluino-mass parameter $M_{3}=3 \mathrm{TeV}$ and the other parts of the spectrum in this scenario (cf. Table 2). In such a case a consistent decoupling of the heavy gluino

\footnotetext{
${ }^{6}$ In [69] a similar effect has been observed. It was found that in case of large gluino mass $m_{\tilde{g}}$ OS renormalization of the top/stop sector (with the physical stop masses used as input) is advantageous over a $\overline{\mathrm{DR}}$ renormalization of the top/stop sector since the $\overline{\mathrm{DR}}$ renormalization leads to terms enhanced in $m_{\tilde{g}}^{2} / M_{S}^{2}$.
} 

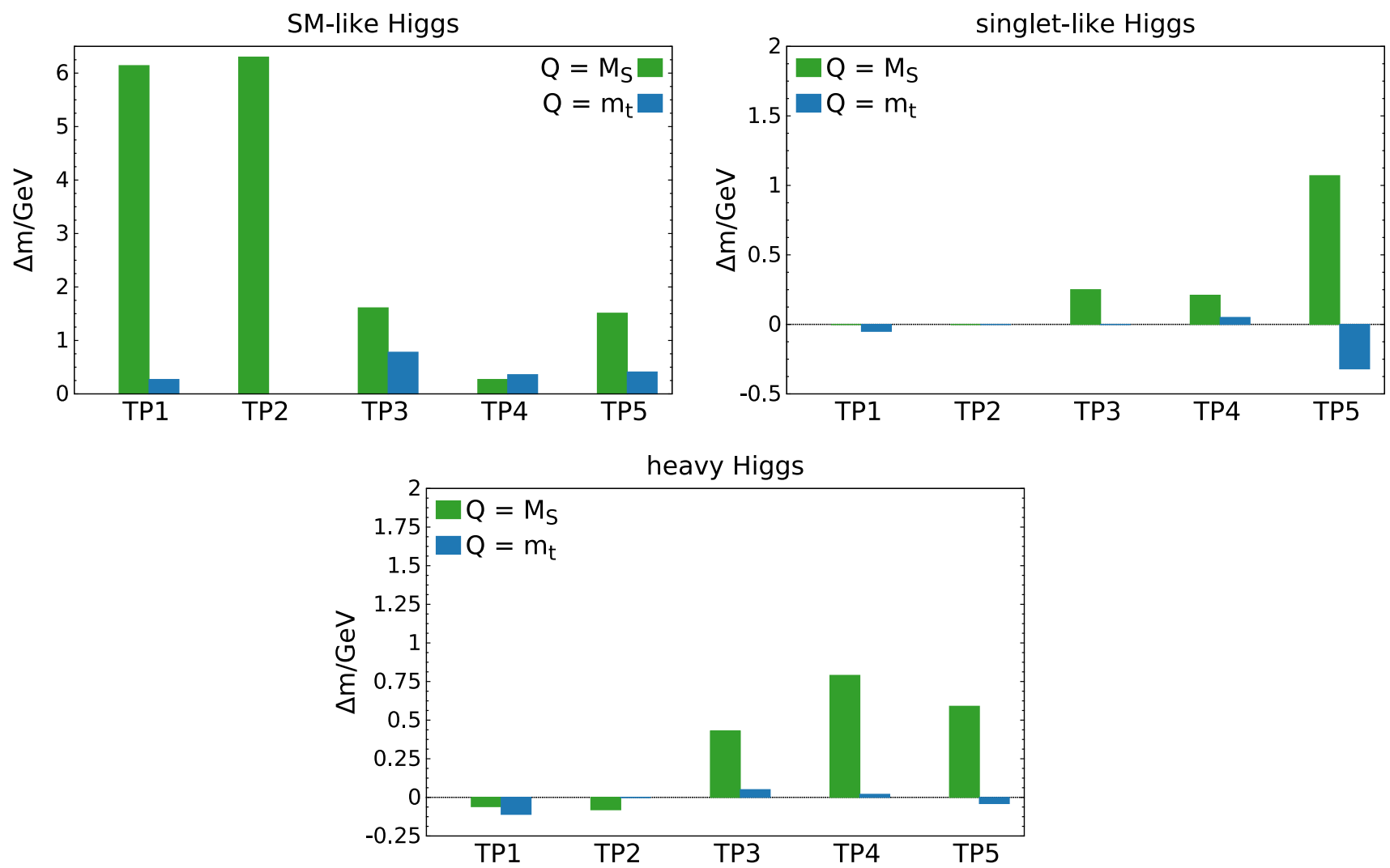

Fig. 2 Difference $\Delta m=M_{h}^{\mathrm{NC}}-M_{h}^{\mathrm{N}-\mathrm{FH}}$ between the predicted Higgs masses of the SM-like, singlet-like and heavy Higgs at the two-loop level calculated at the scales $M_{S}$ and $m_{t}$

should be performed (see the discussion in Ref. [70]), which is beyond the scope of our present analysis. If the heavy gluino is kept in the spectrum for the running from $M_{S}$ to $m_{t}$ and for the conversion from the $\overline{\mathrm{DR}}$ to the OS scheme, the obtained low-scale scenario corresponds to a different physical situation than the high-scale one. The comparison of the results of the high-scale and the low-scale scenario would therefore not describe the difference between the two calculations but rather a difference between two distinct physical situations. We found also that the parameters obtained at $m_{t}$ define a scenario that is highly sensitive to variations of the stop parameters. This is in particular true for $A_{t}$, which at the input scale $M_{S}$ is large compared to the other soft-breaking parameters of the scenario. For example, at the low-scale $m_{t}$, changes of $\left|\Delta A_{t}\right| \approx 100 \mathrm{GeV}$ can yield a change of the mass of the lightest Higgs of $\left|\Delta m_{h_{1}}\right| \approx 2 \mathrm{GeV}$. Since the observed effects are independent of the Higgs-mass calculation itself (see Fig. 1), we regard the scenario TP1 in the present form as not suitable for the discussion of theoretical uncertainties. We will therefore omit this scenario in the following. Note, however, that we explicitly checked that by doing the adjustments of the codes as described in the following sections we find very good agreement between NMSSM-FeynHiggs and NMSSMCALC for both the low- scale and the high-scale scenario of TP1. The results for the low-scale and the high-scale scenario differ by about $3 \mathrm{GeV}$ for the SM-like Higgs in both codes after the adjustments.

\subsection{Renormalization of the electromagnetic coupling constant $\alpha$}

In order to disentangle the effects arising from differences in the renormalization, the coupling constants and the higherorder corrections, we start by comparing the one-loop results for the Higgs-boson masses and mixing matrices. Here we use the versions ' $\mathrm{NC}$ OS' and ' $\mathrm{N}-\mathrm{FH}$ ' as described in the previous section, but restrict the predictions to the pure oneloop contribution to the Higgs-boson self-energies. In this case the only difference between the calculations stems from the different renormalization prescription of the electromagnetic coupling constant $\alpha$, see Table 1 . The corresponding numerical results for the $\mathcal{C P}$-even Higgs-boson masses are given in Table 6.

The differences between the one-loop results applying the different definitions for the electromagnetic coupling constant, shown in Fig. 3 by light green bars, are smaller compared to the observed differences at the two-loop level. They never exceed $1.0 \mathrm{GeV}$ for all masses in all scenarios. In order 
Table 6 One-loop mass predictions for the $\mathcal{C P}$-even scalars for TP2-TP5 when using the indicated versions of NMSSMCALC and NMSSM-FeynHiggs as specified in Sect. 5.3. The mass values of the SM-like scalar are written in bold fonts, those of the singlet-like scalar in italics

\begin{tabular}{|c|c|c|c|c|c|c|c|c|c|}
\hline & \multirow[t]{2}{*}{$Q$} & \multicolumn{2}{|l|}{$\mathrm{TP} 2$} & \multicolumn{2}{|l|}{ TP3 } & \multicolumn{2}{|l|}{ TP4 } & \multicolumn{2}{|l|}{ TP5 } \\
\hline & & $M_{S}$ & $m_{t}$ & $M_{S}$ & $m_{t}$ & $M_{S}$ & $m_{t}$ & $M_{S}$ & $m_{t}$ \\
\hline \multirow[t]{3}{*}{$h_{1}$} & NC OS & 140.67 & - & 90.47 & 90.12 & 132.48 & 134.10 & 120.93 & 120.57 \\
\hline & $\mathrm{N}-\mathrm{FH}$ & 139.68 & - & 90.30 & 89.98 & 132.96 & 133.42 & 120.82 & 120.51 \\
\hline & $\mathrm{N}-\mathrm{FH} \alpha\left(M_{Z}\right)$ & 140.67 & - & 90.38 & 89.96 & 132.73 & 133.22 & 120.91 & 120.53 \\
\hline \multirow[t]{3}{*}{$h_{2}$} & NC OS & 5951.36 & - & 136.35 & 138.25 & 146.45 & 146.84 & 135.56 & 138.27 \\
\hline & $\mathrm{N}-\mathrm{FH}$ & 5951.36 & - & 136.73 & 137.04 & 146.82 & 146.05 & 136.30 & 137.06 \\
\hline & $\mathrm{N}-\mathrm{FH} \alpha\left(M_{Z}\right)$ & 5951.36 & - & 136.74 & 137.19 & 146.59 & 145.89 & 136.32 & 137.26 \\
\hline \multirow[t]{3}{*}{$h_{3}$} & NC OS & 6370.75 & - & 652.80 & 653.13 & 468.63 & 468.32 & 627.34 & 629.19 \\
\hline & $\mathrm{N}-\mathrm{FH}$ & 6370.83 & - & 652.49 & 653.04 & 468.01 & 468.30 & 626.94 & 629.14 \\
\hline & $\mathrm{N}-\mathrm{FH} \alpha\left(M_{Z}\right)$ & 6370.88 & - & 652.62 & 653.18 & 468.37 & 468.60 & 627.08 & 629.23 \\
\hline
\end{tabular}

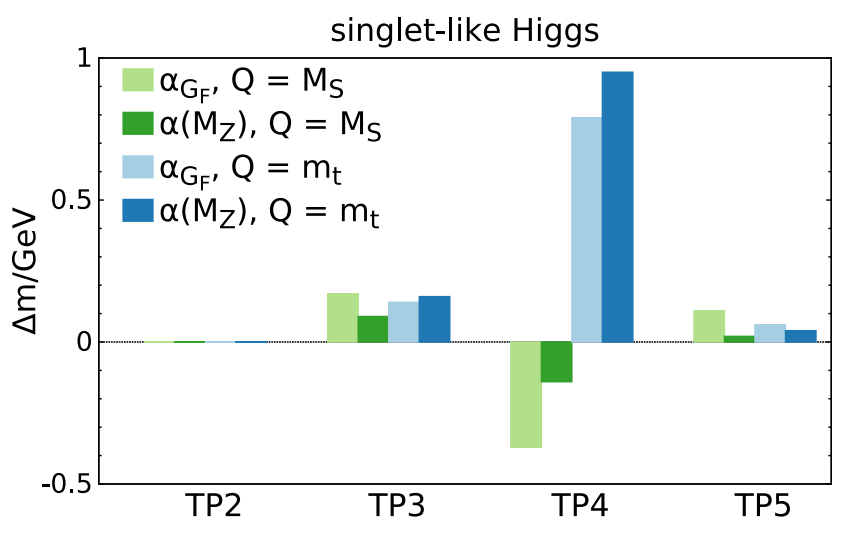

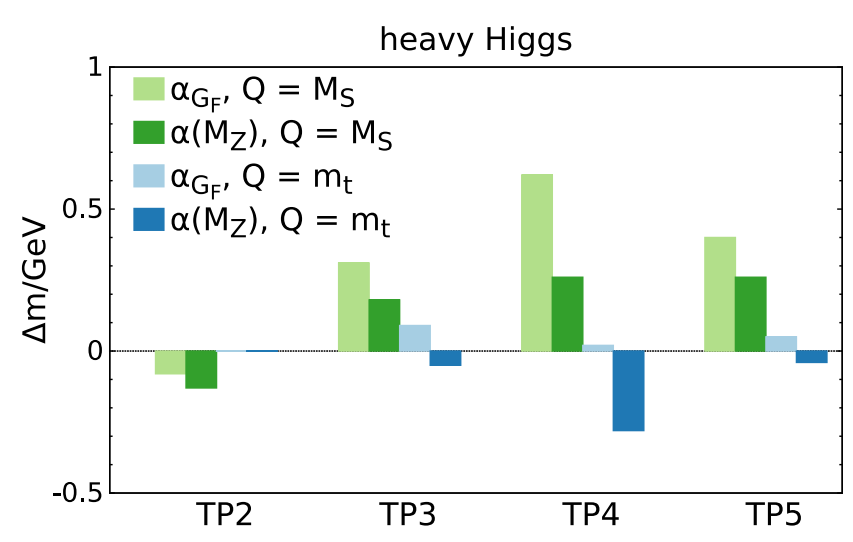

Fig. 3 One-loop difference $\Delta m=M_{h}^{\mathrm{NC}}-M_{h}^{\mathrm{N}-\mathrm{FH}}$ at the scales $M_{S}$ (green) and $m_{t}$ (blue) between NMSSMCALC and NMSSM-FeynHiggs with the reparametrization to $\alpha_{G_{F}}$ (bright) and $\alpha\left(M_{Z}\right)($ dark)

to account for this well-understood difference between the two renormalization schemes for $\alpha_{G_{F}}$ and $\alpha\left(M_{Z}\right)$, which is of the order of unknown electroweak two-loop corrections and can therefore serve as an indication of the possible size of remaining theoretical uncertainties of this type, we now employ a modification of NMSSM-FeynHiggs. In NMSSM-FeynHiggs the treatment of $\alpha$ is a two-step procedure: in the first step a $\overline{\mathrm{DR}}$ reparametrization for the vacuum expectation value $v$ is applied. In the second step this result is then reparametrized in terms of a suitably cho- sen expression for $\alpha$. For the results discussed so far, the electric charge is expressed in terms of the Fermi constant $G_{F}$, the default value in NMSSM-FeynHiggs. As discussed before, this is done to ensure that in NMSSM-FeynHiggs the MSSM limit exactly reproduces the MSSM result of FeynHiggs. For the discussed results the reparametrization of the electromagnetic coupling is only necessary up to the one-loop level, since the two-loop corrections of $\mathcal{O}\left(\alpha_{t} \alpha_{s}\right)$ have been obtained in the gauge-less limit (cf. Ref. [31]). In the following we adjust the second step in the outlined pro- 
Table 7 One-loop mixing-matrix elements for TP2-TP5 when using the indicated versions of NMSSMCALC and NMSSM-FeynHiggs as specified in the text; see Sect. 5.3

\begin{tabular}{|c|c|c|c|c|c|c|c|c|}
\hline \multirow[t]{2}{*}{$i$} & \multirow[t]{2}{*}{$Q$} & & \multicolumn{3}{|l|}{$\mathrm{TP} 2$} & \multicolumn{3}{|l|}{ TP3 } \\
\hline & & & $\left|U_{i 1}^{h}\right|$ & $\left|U_{i 2}^{h}\right|$ & $\left|U_{i 3}^{h}\right|$ & $\left|U_{i 1}^{h}\right|$ & $\left|U_{i 2}^{h}\right|$ & $\left|U_{i 3}^{h}\right|$ \\
\hline \multirow[t]{4}{*}{1} & \multirow[t]{2}{*}{$M_{S}$} & NC OS & 0.1034 & 0.9946 & 0.0004 & 0.2048 & 0.1503 & 0.9672 \\
\hline & & $\mathrm{N}-\mathrm{FH} \alpha\left(M_{Z}\right)$ & 0.1034 & 0.9946 & 0.0004 & 0.1981 & 0.1443 & 0.9695 \\
\hline & \multirow[t]{2}{*}{$m_{t}$} & NC OS & - & - & - & 0.2048 & 0.1553 & 0.9664 \\
\hline & & $\mathrm{N}-\mathrm{FH} \alpha\left(M_{Z}\right)$ & - & - & - & 0.2054 & 0.1601 & 0.9655 \\
\hline \multirow[t]{4}{*}{2} & \multirow[t]{2}{*}{$M_{S}$} & NC OS & 0.0096 & 0.0006 & 1.0000 & 0.2935 & 0.9332 & 0.2071 \\
\hline & & $\mathrm{N}-\mathrm{FH} \alpha\left(M_{Z}\right)$ & 0.0092 & 0.0005 & 1.0000 & 0.2971 & 0.9337 & 0.1997 \\
\hline & \multirow[t]{2}{*}{$m_{t}$} & NC OS & - & - & - & 0.2836 & 0.9356 & 0.2104 \\
\hline & & $\mathrm{N}-\mathrm{FH} \alpha\left(M_{Z}\right)$ & - & - & - & 0.2837 & 0.9344 & 0.2153 \\
\hline \multirow[t]{4}{*}{3} & \multirow[t]{2}{*}{$M_{S}$} & NC OS & 0.9946 & 0.1034 & 0.0096 & 0.9338 & 0.3263 & 0.1470 \\
\hline & & $\mathrm{N}-\mathrm{FH} \alpha\left(M_{Z}\right)$ & 0.9946 & 0.1034 & 0.0092 & 0.9341 & 0.3276 & 0.1421 \\
\hline & \multirow[t]{2}{*}{$m_{t}$} & NC OS & - & - & - & 0.9368 & 0.3172 & 0.1475 \\
\hline & & $\mathrm{N}-\mathrm{FH} \alpha\left(M_{Z}\right)$ & - & - & - & 0.9366 & 0.3182 & 0.1466 \\
\hline \multirow[t]{2}{*}{$i$} & \multirow[t]{2}{*}{$Q$} & & \multicolumn{3}{|l|}{ TP4 } & \multicolumn{3}{|l|}{ TP5 } \\
\hline & & & $\left|U_{i 1}^{h}\right|$ & $\left|U_{i 2}^{h}\right|$ & $\left|U_{i 3}^{h}\right|$ & $\left|U_{i 1}^{h}\right|$ & $\left|U_{i 2}^{h}\right|$ & $\left|U_{i 3}^{h}\right|$ \\
\hline \multirow[t]{4}{*}{1} & \multirow[t]{2}{*}{$M_{S}$} & NC OS & 0.4393 & 0.5717 & 0.6929 & 0.2079 & 0.1294 & 0.9695 \\
\hline & & $\mathrm{N}-\mathrm{FH} \alpha\left(M_{Z}\right)$ & 0.4446 & 0.5948 & 0.6697 & 0.1998 & 0.1211 & 0.9723 \\
\hline & \multirow[t]{2}{*}{$m_{t}$} & $\mathrm{NC} O \mathrm{OS}$ & 0.4422 & 0.6086 & 0.6588 & 0.2117 & 0.1484 & 0.9660 \\
\hline & & $\mathrm{N}-\mathrm{FH} \alpha\left(M_{Z}\right)$ & 0.4538 & 0.6467 & 0.6131 & 0.2151 & 0.1624 & 0.9630 \\
\hline \multirow[t]{4}{*}{2} & \multirow[t]{2}{*}{$M_{S}$} & NC OS & 0.2285 & 0.6749 & 0.7017 & 0.2983 & 0.9356 & 0.1889 \\
\hline & & $\mathrm{N}-\mathrm{FH} \alpha\left(M_{Z}\right)$ & 0.2188 & 0.6529 & 0.7252 & 0.3026 & 0.9362 & 0.1788 \\
\hline & \multirow[t]{2}{*}{$m_{t}$} & NC OS & 0.1982 & 0.6500 & 0.7336 & 0.2832 & 0.9367 & 0.2060 \\
\hline & & $\mathrm{N}-\mathrm{FH} \alpha\left(M_{Z}\right)$ & 0.1730 & 0.6110 & 0.7725 & 0.2812 & 0.9340 & 0.2203 \\
\hline \multirow[t]{4}{*}{3} & \multirow[t]{2}{*}{$M_{S}$} & NC OS & 0.8688 & 0.4666 & 0.1658 & 0.9315 & 0.3285 & 0.1559 \\
\hline & & $\mathrm{N}-\mathrm{FH} \alpha\left(M_{Z}\right)$ & 0.8686 & 0.4689 & 0.1602 & 0.9319 & 0.3300 & 0.1504 \\
\hline & \multirow[t]{2}{*}{$m_{t}$} & NC OS & 0.8747 & 0.4550 & 0.1668 & 0.9354 & 0.3172 & 0.1563 \\
\hline & & $\mathrm{N}-\mathrm{FH} \alpha\left(M_{Z}\right)$ & 0.8742 & 0.4566 & 0.1654 & 0.9352 & 0.3182 & 0.1552 \\
\hline
\end{tabular}

cedure, where we choose to express the electric charge in NMSSM-FeynHiggs by its value $\alpha\left(M_{Z}\right)$, the default value in NMSSMCALC. This modified version is labeled " $\mathrm{N}-\mathrm{FH}$ $\alpha\left(M_{Z}\right)$ ". This modification is expected to yield a better, yet not perfect agreement between the two codes. The remaining difference between results obtained by " $\mathrm{N}-\mathrm{FH} \alpha\left(M_{Z}\right)$ ", where the electric charge is reparametrized to the value $\alpha\left(M_{Z}\right)$, and "NC OS", where the electric charge is renormalized to the value $\alpha\left(M_{Z}\right)$, consists formally also of electroweak corrections of two-loop and higher orders.

The mass predictions for the version " $\mathrm{N}-\mathrm{FH} \alpha\left(M_{Z}\right)$ " are given in Table 6. When compared to the results of "NC OS" at the scale $M_{S}$ the results of the adjusted version "N-FH $\alpha\left(M_{Z}\right)$ " are in better or equally well agreement as the results of the previous version " $\mathrm{N}-\mathrm{FH}$ " without the adjustment. This can be seen by comparing the light and dark green bars in Fig. 3. For the comparison at $Q=m_{t}$, shown as blue bars in Fig. 3, for the SM-like Higgs boson also an improvement is achieved by the reparametrization of $\alpha$ for all scenarios except for TP4, where the agreement is slightly worse. For the other two Higgs bosons the result is less conclusive. In all scenarios but TP4 the reparametrization to $\alpha\left(M_{Z}\right)$ yields an improved or equally well agreement as the results of the version " $\mathrm{N}-\mathrm{FH}$ " without the adjustment at both scales. The mentioned two-loop and higher-order effects from the charge renormalization appear to be more important in the scenario TP4. The mixing-matrix elements, see Table 7, obtained by the two codes agree within $\approx 10 \%$ with the largest differences occurring for the scenarios TP4 and TP5. For the scenario TP4 and the two lighter Higgs states we obtained similar, sizeable values for $\left|U_{i 2}^{h}\right|$ and $\left|U_{i 3}^{h}\right|$ with either code at both scales $M_{S}$ and $m_{t}$, making the assignment of the singlet- and SM-like field ambiguous. We thus follow the identification obtained with the two-loop calculation described in Sect. 5.1. In order to verify that the observed differences between the versions "NC OS" and "N-FH $\alpha\left(M_{Z}\right)$ " are indeed explained by two-loop and higher-order effects from the reparametrization procedure, we compared the predictions of the two versions in the MSSM limit of the TP scenarios. In the MSSM limit, with $\lambda=\kappa \rightarrow 0$, the renormalization constant of $\alpha$ 
Table 8 Mass predictions for the $\mathcal{C} \mathcal{P}$-even scalars for TP2-TP5 when using modified versions of NMSSMCALC in the OS option NC OS (denoted shortly by $\mathrm{NC}$ ) and NMSSM-FeynHiggs (N-FH). Both codes are modified to use an identical numerical value for $\alpha_{s}$. In NMSSM-FeynHiggs the reparametrization to $\alpha\left(M_{Z}\right)\left(\mathrm{N}-\mathrm{FH} \alpha\left(M_{Z}\right)\right)$ is used. The values correspond to the two-loop result obtained with the OS renormalization scheme for the top/stop sectors. The mass values for the SM-like scalar are written in bold fonts, those for the singlet-like scalar in italics

\begin{tabular}{|c|c|c|c|c|c|c|c|c|c|}
\hline & \multirow[t]{2}{*}{$Q$} & \multicolumn{2}{|l|}{$\underline{\text { TP2 }}$} & \multicolumn{2}{|l|}{ TP3 } & \multicolumn{2}{|l|}{ TP4 } & \multicolumn{2}{|l|}{ TP5 } \\
\hline & & $M_{S}$ & $m_{t}$ & $M_{S}$ & $m_{t}$ & $M_{S}$ & $m_{t}$ & $M_{S}$ & $m_{t}$ \\
\hline \multirow[t]{2}{*}{$h_{1}$} & $\mathrm{NC} \alpha_{s} \bmod$ & 114.70 & - & 89.76 & 88.83 & 125.07 & 126.71 & 117.71 & 117.71 \\
\hline & $\mathrm{N}-\mathrm{FH} \alpha\left(M_{Z}\right)$ & 114.65 & - & 89.72 & 89.31 & 125.56 & 125.74 & 118.31 & 117.77 \\
\hline \multirow[t]{2}{*}{$h_{2}$} & $\mathrm{NC} \alpha_{s} \bmod$ & 5951.36 & - & 123.90 & 125.87 & 142.96 & 142.74 & 122.88 & 123.60 \\
\hline & $\mathrm{N}-\mathrm{FH} \alpha\left(M_{Z}\right)$ & 5951.36 & - & 124.26 & 124.88 & 142.98 & 142.59 & 122.81 & 123.08 \\
\hline \multirow[t]{2}{*}{$h_{3}$} & $\mathrm{NC} \alpha_{s} \bmod$ & 6370.76 & - & 652.56 & 652.70 & 467.75 & 467.35 & 627.14 & 628.72 \\
\hline & $\mathrm{N}-\mathrm{FH} \alpha\left(M_{Z}\right)$ & 6370.90 & - & 652.29 & 652.81 & 467.43 & 467.61 & 626.73 & 628.84 \\
\hline
\end{tabular}

drops out as well as the reparametrization. We found that in the MSSM limit there is complete agreement between the two codes at the expected level of numerical accuracy.

The reparametrization to $\alpha\left(M_{Z}\right)$ in NMSSM-FeynHiggs overall yields a better agreement with NMSSMCALC. The effect on the mass prediction for the SM-like Higgs, however, is much smaller than some of the large differences observed for the two-loop mass prediction in Fig. 2. In the subsequent sections a comparison between NMS SMCALC and NMSSM-FeynHiggs, where in the latter code $\alpha$ has been reparametrized to $\alpha\left(M_{Z}\right)$, will be performed at the twoloop level in order to identify the differences that are not caused by the renormalization of the electromagnetic coupling constant $\alpha$.

\subsection{Treatment of the strong coupling constant $\boldsymbol{\alpha}_{s}$}

In the following we analyze the effects of the different treatment of $\alpha_{s}$ in the two codes. In Table 8 the mass predictions at $\mathcal{O}\left(\alpha_{t} \alpha_{s}\right)$ are given for the case that NMSSMCALC is modified such that always the hard-coded $\overline{\mathrm{MS}}$-value of the strong coupling at the scale $m_{t}, \alpha_{s}^{\overline{\mathrm{MS}}}\left(m_{t}\right)=0.10697$ as obtained with the routines of [62], is used. The results of NMSSM-FeynHiggs are not affected by this procedure, since $\alpha_{s} \overline{\mathrm{MS}}\left(m_{t}\right)$ is the standard value that is used. The modified version of NMSSMCALC is labeled "NC $\alpha_{s}$ mod" in the following. As discussed above, for NMSSM-FeynHiggs we continue to use the version reparametrized to $\alpha\left(M_{Z}\right)$. The graphical representation of the differences is depicted in Fig. 4. When both codes use the same numerical value of $\alpha_{s}$ a better agreement with much smaller relative differences between their mass predictions can be observed. The differences between the mass predictions never exceed $1.1 \mathrm{GeV}$, and mostly stay below $0.5 \mathrm{GeV}$ for all masses in all TP scenarios. In the MSSM limit we found again complete agreement between the two codes at the expected level of numerical accuracy now at $\mathcal{O}\left(\alpha_{t} \alpha_{s}\right)$.
We conclude that the main source of the observed difference is the different treatment of the strong coupling constant $\alpha_{S}$ (see Table 1). Although the renormalization prescription and scale dependence of $\alpha_{s}$ represent effects formally of three-loop order, their effects on the Higgs mass predictions can be sizeable. The corresponding mixing-matrix elements are given in Table 9. In the scenarios TP2-TP4 at both scales $M_{S}$ and $m_{t}$ we found differences of less than 5\% for the largest matrix elements, which contain the dominant admixture to the fields $h_{i}$. Larger differences occur for the subleading matrix elements, e.g. in scenario TP3 differences of up to $19 \%$ can be observed. For TP4, subleading matrix elements can differ even by up to a factor of 3 . The matrix elements that differ so strongly between the two codes are, however, only a few percent of the largest one. Even a change of them by a factor of 3 results in relatively small differences compared with the absolute size of the leading matrix elements. For the scenario TP5 we observe larger differences for the largest matrix elements. They differ by up to $20 \%$. The reason why the matrix elements of TP5 show larger discrepancies is the large mixing between the two lightest $\mathcal{C P}$. even Higgs bosons where small changes in the parameters can cause a large effect in the resulting mixing elements. At the scale $M_{S}$ the adapted codes show a better agreement for the largest mixing-matrix elements when compared to their "out-of-the-box" versions (cf. Table 5), where we found differences of up to $\approx 40 \%$. At the scale $m_{t}$ the adapted codes show a worse agreement with differences of up to $\approx 20 \%$ even for the largest matrix elements, while the corresponding difference for the "out-of-the-box" versions never exceeded $3 \%$. For scenario TP5 and each of the adapted codes, however, we find that the mixing-matrix elements can differ by up to $\approx 20 \%$ when evaluated at either the scale $M_{S}$ or $m_{t}$. We conclude that in the scenario TP5 the mixing-matrix elements are very sensitive to small variations of the parameters due to the large mixing between the singlet and SM-like Higgs bosons. The results for the masses are much less sensitive to these effects. 


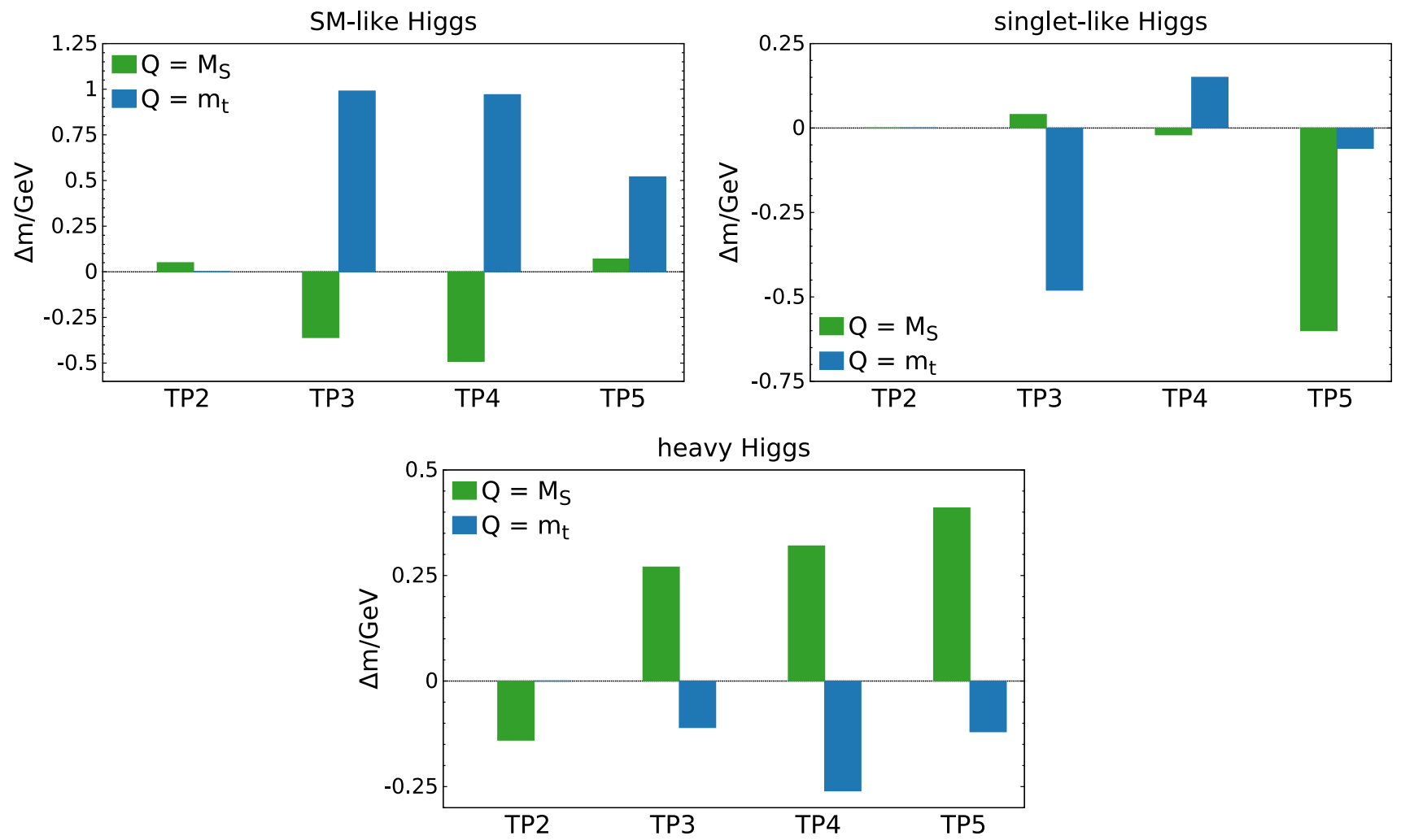

Fig. 4 Difference $\Delta m=M_{h}^{\mathrm{NC}} \alpha_{s}$ mod $-M_{h}^{\mathrm{N}-\mathrm{FH}}$ between the Higgs masses of the SM-like, singlet-like and heavy Higgs at the two-loop level calculated at the scales $M_{S}$ and $m_{t}$

\subsection{MSSM-approximation beyond one-loop in NMSSM-FeynHiggs}

In NMSSM-FeynHiggs the NMSSM contributions beyond one-loop are approximated by the respective corrections from the MSSM at present. This means that at $\mathcal{O}\left(\alpha_{t} \alpha_{s}\right)$ the genuine NMSSM contributions are only incorporated in NMSSMCALC, as will be discussed below. On the other hand, NMSSM-FeynHiggs incorporates further MSSMtype contributions beyond $\mathcal{O}\left(\alpha_{t} \alpha_{s}\right)$. These contributions consist of further leading and subleading two-loop corrections [34,58,71-74] as well as the resummation of large logarithms to all orders for high SUSY mass scales [40,41]. In the MSSM limit it has been found that these corrections can yield $\mathcal{O}(5 \mathrm{GeV})$ corrections in the OS renormalization $[34,40,41,58,71-74]$. This, however, does not take into account the impact of non-zero values of $\lambda$, which have not been evaluated in an OS calculation so far. A $\overline{\mathrm{DR}}$ calculation of the MSSM-approximated $\mathcal{O}\left(\left(\alpha_{t}+\alpha_{b}\right)^{2}\right)$ contributions in [55] for TP1-TP5 gave rise to a $\sim 1 \mathrm{GeV}$ correction (where the corresponding $\mathcal{O}\left(\alpha_{t} \alpha_{s}\right)$ calculation yields somewhat smaller corrections than our OS result), while the genuine NMSSM contributions from the fermion/sfermion and Higgs/Higgsino sectors in the electroweak gauge-less limit [32] gave rise to an additional $\lesssim 1 \mathrm{GeV}$ correction. We leave a more detailed discussion for future work.
At $\mathcal{O}\left(\alpha_{t} \alpha_{s}\right)$ the genuine NMSSM two-loop corrections incorporated in NMSSMCALC give rise to differences to NMSSM-FeynHiggs. In order to estimate their impact we compare the two-loop mass predictions between ' $\mathrm{NC} \alpha_{S}$ mod' and ' $\mathrm{N}-\mathrm{FH} \alpha\left(M_{Z}\right)$ ' given in Table 8. The effect of the MSSM-approximation $\Delta m^{\text {appr }}$ can be obtained by

$\Delta m^{\mathrm{appr}}=\Delta m^{2 \mathrm{~L}}-\Delta m^{1 \mathrm{~L}}$,

where the $\Delta m^{n \mathrm{~L}}$ are the differences between the result of NMSSMCALC and NMSSM-FeynHiggs at the $n$-loop order obtained from the results given in Tables 6 (where we take the 'N-FH $\alpha\left(M_{Z}\right)$ ' value for NMSSM-FeynHiggs) and 8. By this construction the effects of the residual differences arising from the different treatment of the electromagnetic coupling constant $\alpha$ are separated from the effects of the MSSM-approximation. The results of these comparisons are shown in Fig. 5. As expected the approximation has the largest effects for the scenarios TP3-TP5 with large values of $\lambda$. For the SM-like Higgs field $\Delta m_{h}^{\text {appr }}$ does not exceed $\pm 750(500) \mathrm{MeV}$ at the scale $M_{S}\left(m_{t}\right)$, shown as dark green (blue) bars. For the singlet-like Higgs boson it stays below $\pm 750 \mathrm{MeV}$ for both scales, and for the heavy Higgs field we find differences below $\pm 200 \mathrm{MeV}$. This is in accordance with the expected impact of the approximation as described in Ref. [31] as well as with the results of Ref. [33]. 
Table 9 Two-loop mixing-matrix elements for TP2-TP5 when using the indicated versions of NMSSMCALC and NMSSM-FeynHiggs as specified in the text; see

Sect. 5.4

\begin{tabular}{|c|c|c|c|c|c|c|c|c|}
\hline \multirow[t]{2}{*}{$i$} & \multirow[t]{2}{*}{$Q$} & & \multicolumn{3}{|l|}{ TP2 } & \multicolumn{3}{|l|}{ TP3 } \\
\hline & & & $\left|U_{i 1}^{h}\right|$ & $\left|U_{i 2}^{h}\right|$ & $\left|U_{i 3}^{h}\right|$ & $\left|U_{i 1}^{h}\right|$ & $\left|U_{i 2}^{h}\right|$ & $\left|U_{i 3}^{h}\right|$ \\
\hline \multirow[t]{4}{*}{1} & \multirow[t]{2}{*}{$M_{S}$} & $\mathrm{NC} \alpha_{s} \bmod$ & 0.1034 & 0.9946 & 0.0004 & 0.2243 & 0.2140 & 0.9507 \\
\hline & & $\mathrm{N}-\mathrm{FH} \alpha\left(M_{Z}\right)$ & 0.1034 & 0.9946 & 0.0004 & 0.2229 & 0.2270 & 0.9480 \\
\hline & \multirow[t]{2}{*}{$m_{t}$} & $\mathrm{NC} \alpha_{s} \bmod$ & - & - & - & 0.2234 & 0.2204 & 0.9495 \\
\hline & & $\mathrm{N}-\mathrm{FH} \alpha\left(M_{Z}\right)$ & - & - & - & 0.2342 & 0.2610 & 0.9365 \\
\hline \multirow[t]{4}{*}{2} & \multirow[t]{2}{*}{$M_{S}$} & $\mathrm{NC} \alpha_{s} \bmod$ & 0.0096 & 0.0006 & 1.0000 & 0.2756 & 0.9218 & 0.2726 \\
\hline & & $\mathrm{N}-\mathrm{FH} \alpha\left(M_{Z}\right)$ & 0.0092 & 0.0005 & 1.0000 & 0.2751 & 0.9183 & 0.2846 \\
\hline & \multirow[t]{2}{*}{$m_{t}$} & $\mathrm{NC} \alpha_{s} \bmod$ & - & - & - & 0.2660 & 0.9233 & 0.2769 \\
\hline & & $\mathrm{N}-\mathrm{FH} \alpha\left(M_{Z}\right)$ & - & - & - & 0.2566 & 0.9125 & 0.3185 \\
\hline \multirow[t]{4}{*}{3} & \multirow[t]{2}{*}{$M_{S}$} & $\mathrm{NC} \alpha_{s} \bmod$ & 0.9946 & 0.1034 & 0.0096 & 0.9347 & 0.3232 & 0.1478 \\
\hline & & $\mathrm{N}-\mathrm{FH} \alpha\left(M_{Z}\right)$ & 0.9946 & 0.1034 & 0.0092 & 0.9352 & 0.3243 & 0.1423 \\
\hline & \multirow[t]{2}{*}{$m_{t}$} & $\mathrm{NC} \alpha_{s} \bmod$ & - & - & - & 0.9377 & 0.3144 & 0.1476 \\
\hline & & $\mathrm{N}-\mathrm{FH} \alpha\left(M_{Z}\right)$ & - & - & - & 0.9377 & 0.3149 & 0.1467 \\
\hline \multirow[t]{2}{*}{$i$} & \multirow[t]{2}{*}{$Q$} & & \multicolumn{3}{|l|}{ TP4 } & \multicolumn{3}{|l|}{ TP5 } \\
\hline & & & $\left|U_{i 1}^{h}\right|$ & $\left|U_{i 2}^{h}\right|$ & $\left|U_{i 3}^{h}\right|$ & $\left|U_{i 1}^{h}\right|$ & $\left|U_{i 2}^{h}\right|$ & $\left|U_{i 3}^{h}\right|$ \\
\hline \multirow[t]{4}{*}{1} & \multirow[t]{2}{*}{$M_{S}$} & $\mathrm{NC} \alpha_{s} \bmod$ & 0.4839 & 0.7647 & 0.4255 & 0.1417 & 0.7305 & 0.6680 \\
\hline & & $\mathrm{N}-\mathrm{FH} \alpha\left(M_{Z}\right)$ & 0.4872 & 0.8045 & 0.3398 & 0.3319 & 0.6217 & 0.7094 \\
\hline & \multirow[t]{2}{*}{$m_{t}$} & $\mathrm{NC} \alpha_{s} \bmod$ & 0.4766 & 0.7884 & 0.3900 & 0.3382 & 0.6914 & 0.6384 \\
\hline & & $\mathrm{N}-\mathrm{FH} \alpha\left(M_{Z}\right)$ & 0.4779 & 0.8321 & 0.2813 & 0.3476 & 0.7708 & 0.5339 \\
\hline \multirow[t]{4}{*}{2} & \multirow[t]{2}{*}{$M_{S}$} & $\mathrm{NC} \alpha_{s} \bmod$ & 0.0672 & 0.4524 & 0.8893 & 0.3315 & 0.6008 & 0.7274 \\
\hline & & $\mathrm{N}-\mathrm{FH} \alpha\left(M_{Z}\right)$ & 0.0270 & 0.3751 & 0.9266 & 0.1379 & 0.7120 & 0.6885 \\
\hline & \multirow[t]{2}{*}{$m_{t}$} & $\mathrm{NC} \alpha_{s} \bmod$ & 0.0413 & 0.4219 & 0.9057 & 0.0922 & 0.6508 & 0.7536 \\
\hline & & $\mathrm{N}-\mathrm{FH} \alpha\left(M_{Z}\right)$ & 0.0122 & 0.3266 & 0.9451 & 0.0482 & 0.5540 & 0.8311 \\
\hline \multirow[t]{4}{*}{3} & \multirow[t]{2}{*}{$M_{S}$} & $\mathrm{NC} \alpha_{s} \bmod$ & 0.8726 & 0.4589 & 0.1675 & 0.9327 & 0.3245 & 0.1570 \\
\hline & & $\mathrm{N}-\mathrm{FH} \alpha\left(M_{Z}\right)$ & 0.8729 & 0.4606 & 0.1611 & 0.9332 & 0.3264 & 0.1506 \\
\hline & \multirow[t]{2}{*}{$m_{t}$} & $\mathrm{NC} \alpha_{s} \bmod$ & 0.8782 & 0.4477 & 0.1685 & 0.9365 & 0.3138 & 0.1564 \\
\hline & & $\mathrm{N}-\mathrm{FH} \alpha\left(M_{Z}\right)$ & 0.8783 & 0.4482 & 0.1662 & 0.9364 & 0.3147 & 0.1554 \\
\hline
\end{tabular}

\subsection{Comparison with $\overline{\mathbf{D R}}$ calculation in NMSSMCALC}

As a final step we now compare between different renormalization schemes. For all our results shown up to now we have used an OS renormalization of the parameters in the top/stop sector. NMSSMCALC offers, however, also the possibility to switch between OS and $\overline{\mathrm{DR}}$ renormalization of the top/stop sector, which affects the $\mathcal{O}\left(\alpha_{t} \alpha_{S}\right)$ corrections. In this section the default value of NMSSMCALC for $\alpha_{s}$ in the $\overline{\mathrm{DR}}$ scheme at the scale $Q$ is used.

In Table 10 the predictions for the neutral Higgs boson masses from NMSSMCALC with OS renormalization (first line) and with $\overline{\mathrm{DR}}$ renormalization (second line) of the top/stop sector are given for TP2-TP5. ${ }^{7}$ The numbers corresponding to the OS renormalization of the top/stop sector in Table 10 are identical to the NMSSMCALC results in Table 4. The differences in the Higgs masses due to the change of the

\footnotetext{
$\overline{7}$ Note that wherever we give values at the scale $Q=m_{t}$ the numerical value of the scale is taken to be the top pole mass, $m_{t}=172.9 \mathrm{GeV}$.
}

renormalization scheme between top/stop sector are visualized in Fig. 6. The values of the stop and top masses in the $\overline{\mathrm{DR}}$ scheme can be found in Table 11, the stop masses as obtained in the OS scheme in Table 3.

As can be inferred from Table 10 and Fig. 6, the different renormalization schemes lead in general to differences of $\mathcal{O}(1 \mathrm{GeV})$ for the SM-like and singlet-like Higgs boson with a maximum difference of $1.9 \mathrm{GeV}$ for the SM-like Higgs boson. For the heavy Higgs bosons the maximum difference reaches up to $0.5 \mathrm{GeV}$. The effects are of similar size for both the scale $Q=M_{S}$ and $Q=m_{t}$ and most strongly pronounced for the SM-like Higgs boson, which is affected most by the corrections of the top/stop sector as it has the largest $\phi_{2}$ component, the component that couples to uptype quarks. The numerical differences between the different renormalization schemes are indicative of the theoretical uncertainties due to the missing higher-order corrections of $\mathcal{O}\left(\alpha_{t} \alpha_{s}^{2}\right)$. However, one should keep in mind that in Sect. 5.4 we found that using a different $\alpha_{s}$ has a larger impact on the Higgs boson mass than the difference due to different renor- 

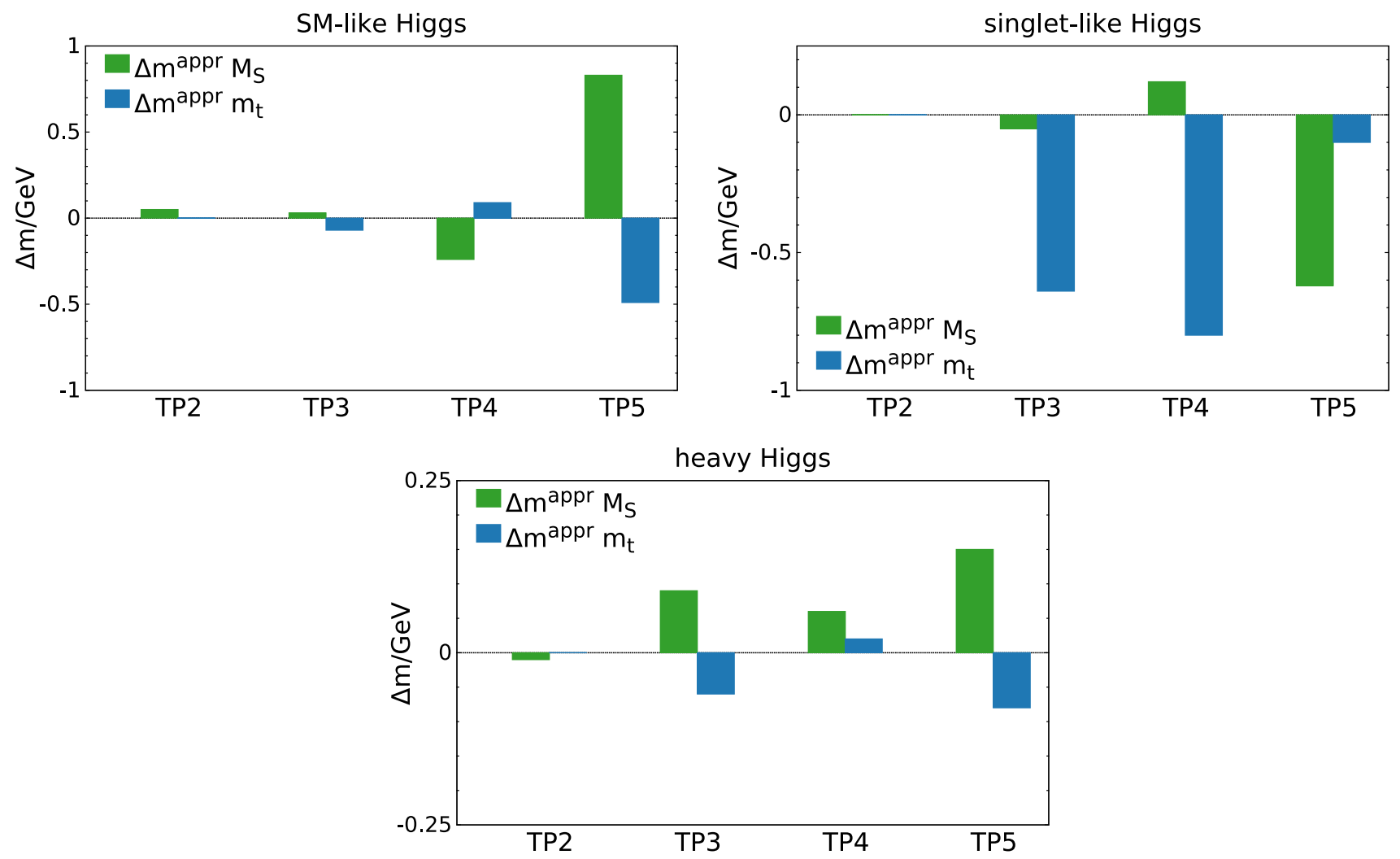

Fig. $5 \Delta m^{\text {appr }}=\Delta m^{2 \mathrm{~L}}-\Delta m^{1 \mathrm{~L}}$ : size of the effect of the MSSM-approximation employed at $\mathcal{O}\left(\alpha_{t} \alpha_{s}\right)$ in NMSSM-FeynHiggs. The used version of NMSSM-FeynHiggs employs the reparametrization to $\alpha\left(M_{Z}\right)$

Table 10 Mass predictions for the $\mathcal{C P}$-even scalars for TP2-TP5 when using the OS and $\overline{\mathrm{DR}}$ renormalization in the top/stop sector. The mass values of the SM-like scalar are written in bold fonts, those of the singlet-like scalar in italics

\begin{tabular}{|c|c|c|c|c|c|c|c|c|c|}
\hline & \multirow[t]{2}{*}{$Q$} & \multicolumn{2}{|l|}{ TP2 } & \multicolumn{2}{|l|}{ TP3 } & \multicolumn{2}{|l|}{ TP4 } & \multicolumn{2}{|l|}{ TP5 } \\
\hline & & $M_{S}$ & $m_{t}$ & $M_{S}$ & $m_{t}$ & $M_{S}$ & $m_{t}$ & $M_{S}$ & $m_{t}$ \\
\hline \multirow[t]{2}{*}{$h_{1}$} & NC OS & 120.42 & - & 89.92 & 88.81 & 126.44 & 126.65 & 119.54 & 117.63 \\
\hline & $\mathrm{NC} \overline{\mathrm{DR}}$ & 118.57 & - & 90.17 & 88.94 & 126.15 & 125.90 & 119.86 & 118.56 \\
\hline \multirow[t]{2}{*}{$h_{2}$} & NC OS & 5951.36 & - & 126.16 & 125.80 & 143.32 & 142.73 & 124.44 & 123.51 \\
\hline & $\mathrm{NC} \overline{\mathrm{DR}}$ & 5951.36 & - & 124.88 & 124.86 & 142.38 & 142.16 & 123.28 & 125.20 \\
\hline \multirow[t]{2}{*}{$h_{3}$} & NC OS & 6370.77 & - & 652.60 & 652.70 & 467.89 & 467.35 & 627.18 & 628.72 \\
\hline & $\mathrm{NC} \overline{\mathrm{DR}}$ & 6371.21 & - & 652.44 & 652.65 & 467.39 & 467.03 & 626.97 & 628.75 \\
\hline
\end{tabular}

malization schemes of the top/stop sector. Since the scale choice of $\alpha_{s}$ is formally a higher-order effect this points to a larger higher-order uncertainty than the one we obtain here. The same observation, namely that, from different treatments of $\alpha_{s}$, one obtains a less optimistic result for the theoretical uncertainty than by a comparison between $\overline{\mathrm{DR}}$ and OS renormalization scheme, was also made in Ref. [69] in the context of the MSSM with Dirac gluinos.

In Table 12 the mixing-matrix elements for the different options of the renormalization of the top/stop sector can be found. For TP2 the renormalization scheme has basically no influence on the mixing-matrix elements. For TP3 the influence of the renormalization scheme is well below $2 \%$, whereas for TP4 and TP5 in some cases the renormalization scheme can change the mixing-matrix elements by more than a factor 2. These large differences occur in the smallest mixing-matrix element of the respective Higgs boson for the singlet-like Higgs as well as for a SM-like Higgs with sizeable singlet admixture. For most of the matrix elements the change due to the renormalization scheme is, however, well below $10 \%$.

Finally, we also want to make contact with the discussion in Ref. [55]. Contrary to our scenarios, where we used $M_{H^{ \pm}}$ as input, in Ref. [55] $A_{\lambda}$ was used as input. This corresponds to a slightly different renormalization scheme in NMSSMCALC. If $M_{H^{ \pm}}$is used as input, the charged Higgs mass is 

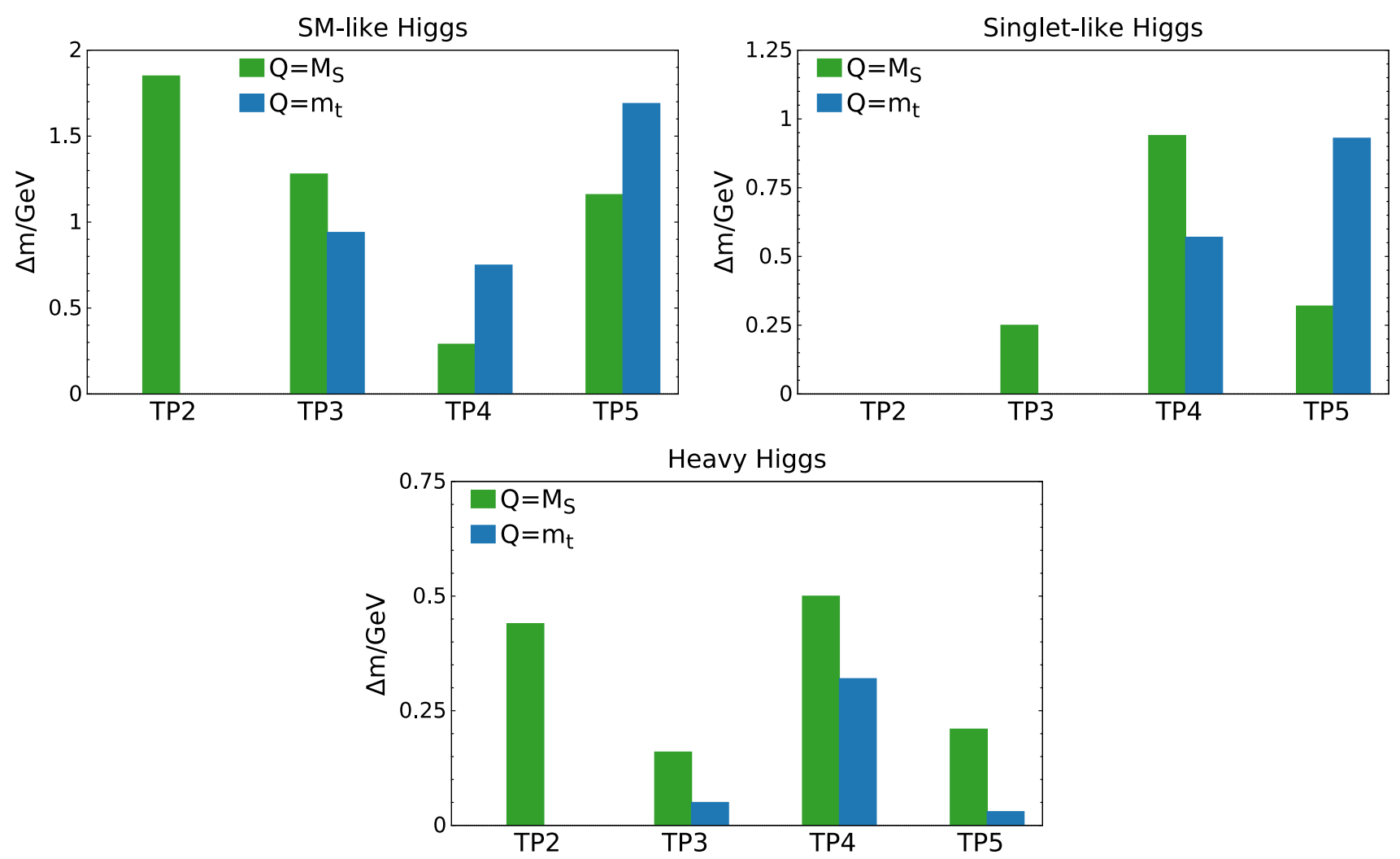

Fig. 6 Absolute difference $\Delta m=\mid M_{h}^{\mathrm{NC}}$ OS $-M_{h}^{\mathrm{NC}} \overline{\mathrm{DR}} \mid$ from Table 10. For TP2 higher-order corrections to the mass of the singlet-like field are suppressed due to the small value of $\lambda$

Table $11 \overline{\mathrm{DR}}$ top and stop masses, given in GeV, in the TP2-TP5 scenarios obtained by the routines of NMSSMCALC. The shift from the top-quark pole mass to the $\overline{\mathrm{DR}}$ top-quark mass $m_{t}^{\overline{\mathrm{DR}}}$ is performed as outlined in Ref. [33], taking into account QCD corrections up to $\mathcal{O}\left(\alpha_{s}^{2}\right)$

\begin{tabular}{|c|c|c|c|c|c|c|c|c|}
\hline \multirow[t]{2}{*}{$Q$} & \multicolumn{2}{|l|}{ TP2 } & \multicolumn{2}{|l|}{ TP3 } & \multicolumn{2}{|l|}{ TP4 } & \multicolumn{2}{|l|}{ TP5 } \\
\hline & $M_{S}$ & $m_{t}$ & $M_{S}$ & $m_{t}$ & $M_{S}$ & $m_{t}$ & $M_{S}$ & $m_{t}$ \\
\hline$m_{t}^{\overline{\mathrm{DR}}}$ & 136.7 & - & 143.9 & 154.2 & 146.9 & 155.8 & 140.0 & 152. \\
\hline$m_{\tilde{t}_{1}}^{\overline{\mathrm{DR}}}$ & 488.4 & - & 940.8 & 1195 & 671.8 & 839.1 & 1503 & 1548 \\
\hline$m_{\tilde{t}_{2}}^{\overline{\mathrm{DR}}}$ & 2509 & - & 1074 & 1267 & 845.3 & 949.7 & 1509 & 1591 \\
\hline
\end{tabular}

renormalized OS and subsequently $A_{\lambda}$ is determined from the charged Higgs mass, whereas if $A_{\lambda}$ is given as input it is renormalized $\overline{\mathrm{DR}}$. In Table 13 we show values where the input is given by $A_{\lambda}$ (first line) and by $M_{H^{ \pm}}$(second line). All values in Table 13 are given for the $\overline{\mathrm{DR}}$ renormalization scheme of the top/stop sector. The first line corresponds to the "out-of-the-box" NMSSMCALC values as given in Ref. [55]. The effect of the way $A_{\lambda}$ (or, respectively, $M_{H^{ \pm}}$) is renormalized is small. Only for the most singlet-like Higgs boson it can exceed $1 \mathrm{GeV}$. For all the other Higgs bosons it is always well below $1 \mathrm{GeV}$, and in particular for the SM-like Higgs boson it is at the level of $\mathcal{O}(100 \mathrm{MeV})$. In Table 14 the values of the mixing-matrix elements are given. Like for the Higgs masses the differences between the input $A_{\lambda}$ or $M_{H^{ \pm}}$ is small. It should finally be noted that if $M_{H^{ \pm}}$is input in NMSSMCALC the $A_{\lambda}$ in the output file is determined from $M_{H^{ \pm}}$at tree level. This implies that the $A_{\lambda}$ in the output of the computation with input $M_{H^{ \pm}}$will differ from the $A_{\lambda}$ given in Ref. [55].

\section{Conclusions}

We have analyzed the predictions for the Higgs-boson masses and mixing matrices in the NMSSM based on an OS renormalization of the top/scalar top sector. We compared the implementation of the results obtained in this scheme in the codes NMSSMCALC and NMSSM-FeynHiggs up to $\mathcal{O}\left(\alpha_{t} \alpha_{s}\right)$ (omitting further MSSM-like higher-order corrections implemented in NMSSM-FeynHiggs). Differences in the calculations implemented in the two codes arise from different renormalization prescriptions and different treatments of the electromagnetic and strong coupling constants, which provide an indication of the possible size of unknown higherorder corrections. Furthermore genuine NMSSM corrections of $\mathcal{O}\left(\alpha_{t} \alpha_{s}\right)$ are implemented in NMSSMCALC, and from the comparison with NMSSM-FeynHiggs one can infer the rel- 
Table 12 Absolute values for the mixing-matrix elements of the $\mathcal{C P}$-even scalar sector for TP2-TP5 when using the OS or the $\overline{\mathrm{DR}}$ renormalization of the top/stop sector within NMSSMCALC
Table 13 Mass predictions for the $\mathcal{C P}$-even scalars for TP2-TP5 when using the $\overline{\mathrm{DR}}$ renormalization in the top/stop sector for either $A_{\lambda}$ as input or $M_{H^{ \pm}}$. The mass values of the SM-like scalar are written in bold fonts, those of the singlet-like scalar in italics

\begin{tabular}{|c|c|c|c|c|c|c|c|c|}
\hline \multirow[t]{2}{*}{$i$} & \multirow[t]{2}{*}{$Q$} & & \multicolumn{3}{|l|}{ TP2 } & \multicolumn{3}{|l|}{ TP3 } \\
\hline & & & $\left|U_{i 1}^{h}\right|$ & $\left|U_{i 2}^{h}\right|$ & $\left|U_{i 3}^{h}\right|$ & $\left|U_{i 1}^{h}\right|$ & $\left|U_{i 2}^{h}\right|$ & $\left|U_{i 3}^{h}\right|$ \\
\hline \multirow[t]{4}{*}{1} & \multirow[t]{2}{*}{$M_{S}$} & NC OS & 0.1034 & 0.9946 & 0.0004 & 0.2199 & 0.1994 & 0.9549 \\
\hline & & $\mathrm{NC} \overline{\mathrm{DR}}$ & 0.1034 & 0.9946 & 0.0004 & 0.2189 & 0.1962 & 0.9558 \\
\hline & \multirow[t]{2}{*}{$m_{t}$} & NC OS & - & - & - & 0.2236 & 0.2210 & 0.9493 \\
\hline & & $\mathrm{NC} \overline{\mathrm{DR}}$ & - & - & - & 0.2237 & 0.2221 & 0.9490 \\
\hline \multirow[t]{4}{*}{2} & \multirow[t]{2}{*}{$M_{S}$} & NC OS & 0.0096 & 0.0006 & 1.0000 & 0.2797 & 0.9249 & 0.2575 \\
\hline & & $\mathrm{NC} \overline{\mathrm{DR}}$ & 0.0095 & 0.0006 & 1.0000 & 0.2802 & 0.9257 & 0.2542 \\
\hline & \multirow[t]{2}{*}{$m_{t}$} & NC OS & - & - & - & 0.2659 & 0.9232 & 0.2775 \\
\hline & & $\mathrm{NC} \overline{\mathrm{DR}}$ & - & - & - & 0.2656 & 0.9230 & 0.2786 \\
\hline \multirow[t]{4}{*}{3} & \multirow[t]{2}{*}{$M_{S}$} & NC OS & 0.9946 & 0.1034 & 0.0096 & 0.9346 & 0.3237 & 0.1476 \\
\hline & & $\mathrm{NC} \overline{\mathrm{DR}}$ & 0.9946 & 0.1034 & 0.0095 & 0.9346 & 0.3235 & 0.1476 \\
\hline & \multirow[t]{2}{*}{$m_{t}$} & $\mathrm{NC}$ OS & - & - & - & 0.9377 & 0.3144 & 0.1476 \\
\hline & & $\mathrm{NC} \overline{\mathrm{DR}}$ & - & - & - & 0.9378 & 0.3144 & 0.1475 \\
\hline \multirow[t]{2}{*}{$i$} & \multirow[t]{2}{*}{$Q$} & & \multicolumn{3}{|l|}{ TP4 } & \multicolumn{3}{|l|}{ TP5 } \\
\hline & & & $\left|U_{i 1}^{h}\right|$ & $\left|U_{i 2}^{h}\right|$ & $\left|U_{i 3}^{h}\right|$ & $\left|U_{i 1}^{h}\right|$ & $\left|U_{i 2}^{h}\right|$ & $\left|U_{i 3}^{h}\right|$ \\
\hline \multirow[t]{4}{*}{1} & \multirow[t]{2}{*}{$M_{S}$} & NC OS & 0.4813 & 0.7432 & 0.4648 & 0.2845 & 0.3943 & 0.8738 \\
\hline & & $\mathrm{NC} \overline{\mathrm{DR}}$ & 0.4863 & 0.7790 & 0.3959 & 0.2994 & 0.4536 & 0.8394 \\
\hline & \multirow[t]{2}{*}{$m_{t}$} & NC OS & 0.4766 & 0.7886 & 0.3885 & 0.3393 & 0.6991 & 0.6294 \\
\hline & & $\mathrm{NC} \overline{\mathrm{DR}}$ & 0.4777 & 0.8098 & 0.3407 & 0.3114 & 0.5379 & 0.7834 \\
\hline \multirow[t]{4}{*}{2} & \multirow[t]{2}{*}{$M_{S}$} & NC OS & 0.0895 & 0.4858 & 0.8694 & 0.2224 & 0.8594 & 0.4603 \\
\hline & & $\mathrm{NC} \overline{\mathrm{DR}}$ & 0.0515 & 0.4267 & 0.9029 & 0.2017 & 0.8298 & 0.5203 \\
\hline & \multirow[t]{2}{*}{$m_{t}$} & NC OS & 0.0411 & 0.4215 & 0.9069 & 0.0882 & 0.6425 & 0.7612 \\
\hline & & $\mathrm{NC} \overline{\mathrm{DR}}$ & 0.0162 & 0.3796 & 0.9250 & 0.1623 & 0.7822 & 0.6015 \\
\hline \multirow[t]{4}{*}{3} & \multirow[t]{2}{*}{$M_{S}$} & NC OS & 0.8720 & 0.4600 & 0.1673 & 0.9325 & 0.3253 & 0.1568 \\
\hline & & $\mathrm{NC} \overline{\mathrm{DR}}$ & 0.8723 & 0.4595 & 0.1674 & 0.9326 & 0.3251 & 0.1569 \\
\hline & \multirow[t]{2}{*}{$m_{t}$} & NC OS & 0.8782 & 0.4477 & 0.1685 & 0.9365 & 0.3137 & 0.1564 \\
\hline & & $\mathrm{NC} \overline{\mathrm{DR}}$ & 0.8784 & 0.4474 & 0.1682 & 0.9363 & 0.3144 & 0.1563 \\
\hline
\end{tabular}

\begin{tabular}{|c|c|c|c|c|c|c|c|c|c|}
\hline & \multirow[t]{2}{*}{$Q$} & \multicolumn{2}{|l|}{ TP2 } & \multicolumn{2}{|l|}{ TP3 } & \multicolumn{2}{|l|}{ TP4 } & \multicolumn{2}{|l|}{ TP5 } \\
\hline & & $M_{S}$ & $m_{t}$ & $M_{S}$ & $m_{t}$ & $M_{S}$ & $m_{t}$ & $M_{S}$ & $m_{t}$ \\
\hline \multirow[t]{2}{*}{$h_{1}$} & $\mathrm{NC} A_{\lambda}$ & 118.57 & - & 90.88 & 87.78 & 126.37 & 125.76 & 120.32 & 118.65 \\
\hline & NC $M_{H^{ \pm}}$ & 118.57 & - & 90.17 & 88.94 & 126.15 & 125.90 & 119.86 & 118.56 \\
\hline \multirow[t]{2}{*}{$h_{2}$} & $\mathrm{NC} A_{\lambda}$ & 5951.36 & - & 124.86 & 124.68 & 142.59 & 141.28 & 123.14 & 125.26 \\
\hline & NC $M_{H^{ \pm}}$ & 5951.36 & - & 124.88 & 124.86 & 142.38 & 142.16 & 123.28 & 125.20 \\
\hline \multirow[t]{2}{*}{$h_{3}$} & $\mathrm{NC} A_{\lambda}$ & 6371.31 & - & 652.48 & 652.64 & 467.42 & 467.01 & 627.00 & 628.77 \\
\hline & $\mathrm{NC} M_{H^{ \pm}}$ & 6371.21 & - & 652.44 & 652.65 & 467.39 & 467.03 & 626.97 & 628.75 \\
\hline
\end{tabular}

evance of these corrections. As a final step, going beyond the OS prescription in the top/sector, also a comparison with the $\overline{\mathrm{DR}}$ renormalization as implemented in NMSSMCALC has been performed. Our work complements and extends the results obtained in Ref. [55], where the Higgs-boson mass calculations in different $\overline{\mathrm{DR}}$ codes had been compared. In order to make contact with this analysis, we employed the same scenarios (TP2-TP5) as in [55]. (The scenarios TP1 and TP6 have found not to be useful for our com- parison of NMSSMCALC and NMSSM-FeynHiggs.) The scenarios are defined at the stop mass scale $M_{S}$. Since diagrammatic calculations as implemented in NMSSMCALC and NMSSM-FeynHiggs are in general designed to evaluate the Higgs-boson sector for SUSY scales that are not widely separated from the weak scale, we also evolved the TP scenarios down to the scale of the top-quark mass. All Higgs mass evaluations have been done at these two scales. At both scales the original $\overline{\mathrm{DR}}$ parameters have been converted to OS parame- 
Table 14 Absolute values for the mixing-matrix elements of the $\mathcal{C P}$-even scalar sector for TP2-TP5 when using the $\overline{\mathrm{DR}}$ renormalization of the top/stop sector for either $A_{\lambda}$ as input or $M_{H^{ \pm}}$

\begin{tabular}{|c|c|c|c|c|c|c|c|c|}
\hline \multirow[t]{2}{*}{$i$} & \multirow[t]{2}{*}{$Q$} & & \multicolumn{3}{|l|}{ TP2 } & \multicolumn{3}{|l|}{ TP3 } \\
\hline & & & $\left|U_{i 1}^{h}\right|$ & $\left|U_{i 2}^{h}\right|$ & $\left|U_{i 3}^{h}\right|$ & $\left|U_{i 1}^{h}\right|$ & $\left|U_{i 2}^{h}\right|$ & $\left|U_{i 3}^{h}\right|$ \\
\hline \multirow[t]{4}{*}{1} & \multirow[t]{2}{*}{$M_{S}$} & $\mathrm{NC} A_{\lambda}$ & 0.1034 & 0.9946 & 0.0004 & 0.2177 & 0.1923 & 0.9569 \\
\hline & & $\mathrm{NC} M_{H^{ \pm}}$ & 0.1034 & 0.9946 & 0.0004 & 0.2189 & 0.1962 & 0.9558 \\
\hline & \multirow[t]{2}{*}{$m_{t}$} & $\mathrm{NC} A_{\lambda}$ & - & - & - & 0.2212 & 0.2142 & 0.9514 \\
\hline & & NC $M_{H^{ \pm}}$ & - & - & - & 0.2237 & 0.2221 & 0.9490 \\
\hline \multirow[t]{4}{*}{2} & \multirow[t]{2}{*}{$M_{S}$} & $\mathrm{NC} A_{\lambda}$ & 0.0095 & 0.0006 & 1.0000 & 0.2811 & 0.9265 & 0.2502 \\
\hline & & NC $M_{H^{ \pm}}$ & 0.0095 & 0.0006 & 1.0000 & 0.2802 & 0.9257 & 0.2542 \\
\hline & \multirow[t]{2}{*}{$m_{t}$} & $\mathrm{NC} A_{\lambda}$ & - & - & - & 0.2677 & 0.9248 & 0.2705 \\
\hline & & $\mathrm{NC} M_{H^{ \pm}}$ & - & - & - & 0.2656 & 0.9230 & 0.2786 \\
\hline \multirow[t]{4}{*}{3} & \multirow[t]{2}{*}{$M_{S}$} & $\mathrm{NC} A_{\lambda}$ & 0.9946 & 0.1034 & 0.0095 & 0.9347 & 0.3234 & 0.1476 \\
\hline & & $\mathrm{NC} M_{H^{ \pm}}$ & 0.9946 & 0.1034 & 0.0095 & 0.9346 & 0.3235 & 0.1476 \\
\hline & \multirow[t]{2}{*}{$m_{t}$} & $\mathrm{NC} A_{\lambda}$ & - & - & - & 0.9378 & 0.3145 & 0.1472 \\
\hline & & NC $M_{H^{ \pm}}$ & - & - & - & 0.9378 & 0.3144 & 0.1475 \\
\hline \multirow[t]{2}{*}{$i$} & \multirow[t]{2}{*}{$Q$} & & \multicolumn{3}{|l|}{ TP4 } & \multicolumn{3}{|l|}{ TP5 } \\
\hline & & & $\left|U_{i 1}^{h}\right|$ & $\left|U_{i 2}^{h}\right|$ & $\left|U_{i 3}^{h}\right|$ & $\left|U_{i 1}^{h}\right|$ & $\left|U_{i 2}^{h}\right|$ & $\left|U_{i 3}^{h}\right|$ \\
\hline \multirow[t]{4}{*}{1} & \multirow[t]{2}{*}{$M_{S}$} & $\mathrm{NC} A_{\lambda}$ & 0.4869 & 0.7849 & 0.3832 & 0.2967 & 0.4433 & 0.8459 \\
\hline & & NC $M_{H^{ \pm}}$ & 0.4863 & 0.7790 & 0.3959 & 0.2994 & 0.4536 & 0.8394 \\
\hline & \multirow[t]{2}{*}{$m_{t}$} & $\mathrm{NC} A_{\lambda}$ & 0.4774 & 0.8045 & 0.3534 & 0.3129 & 0.5451 & 0.7778 \\
\hline & & $\mathrm{NC} M_{H^{ \pm}}$ & 0.4777 & 0.8098 & 0.3407 & 0.3114 & 0.5379 & 0.7834 \\
\hline \multirow[t]{4}{*}{2} & \multirow[t]{2}{*}{$M_{S}$} & $\mathrm{NC} A_{\lambda}$ & 0.0447 & 0.4158 & 0.9084 & 0.2055 & 0.8354 & 0.5099 \\
\hline & & $\mathrm{NC} M_{H^{ \pm}}$ & 0.0515 & 0.4267 & 0.9029 & 0.2017 & 0.8298 & 0.5203 \\
\hline & \multirow[t]{2}{*}{$m_{t}$} & $\mathrm{NC} A_{\lambda}$ & 0.0232 & 0.3906 & 0.9203 & 0.1594 & 0.7772 & 0.6088 \\
\hline & & $\mathrm{NC} M_{H^{ \pm}}$ & 0.0162 & 0.3796 & 0.9250 & 0.1623 & 0.7822 & 0.6015 \\
\hline \multirow[t]{4}{*}{3} & \multirow[t]{2}{*}{$M_{S}$} & $\mathrm{NC} A_{\lambda}$ & 0.8723 & 0.4594 & 0.1674 & 0.9326 & 0.3251 & 0.1568 \\
\hline & & NC $M_{H^{ \pm}}$ & 0.8723 & 0.4595 & 0.1674 & 0.9326 & 0.3251 & 0.1569 \\
\hline & \multirow[t]{2}{*}{$m_{t}$} & $\mathrm{NC} A_{\lambda}$ & 0.8784 & 0.4476 & 0.1678 & 0.9363 & 0.3144 & 0.1563 \\
\hline & & $\mathrm{NC} M_{H^{ \pm}}$ & 0.8784 & 0.4474 & 0.1682 & 0.9363 & 0.3144 & 0.1563 \\
\hline
\end{tabular}

ters that were subsequently used as input for NMSSMCALC and NMSSM-FeynHiggs.

We started with an "out-of-the-box" comparison of the two codes and found large differences of several $\mathrm{GeV}$ between the two codes. In order to disentangle the origin of the differences we first concentrated on the one-loop results. While at the one-loop level both codes perform a complete calculation, they differ in the renormalization of the electromagnetic coupling constant $\alpha$. The resulting differences are formally of electroweak two-loop order. For the further comparison these differences, which yield an indication of the possible size of unknown higher-order corrections of this type, have been adjusted by reparametrizing NMSSM-FeynHiggs to the value used by NMSSMCALC. In a second step, in the two-loop $\mathcal{O}\left(\alpha_{t} \alpha_{s}\right)$ corrections we adjusted the strong coupling constant $\alpha_{S}$ in NMSSMCALC, where $\alpha_{s}^{\overline{\mathrm{DR}}}(Q)$ is employed, to $\alpha_{s}^{\overline{\mathrm{MS}}}\left(m_{t}\right)$ as used by NMSSM-FeynHiggs. Although this difference is formally only of three-loop order, this change improved the agreement between the two codes by several $\mathrm{GeV}$ for the cases where large discrepancies had been observed. The remaining differences of $\mathcal{O}(0.5 \mathrm{GeV})$ are due to the genuine NMSSM corrections in the $\mathcal{O}\left(\alpha_{t} \alpha_{S}\right)$ corrections that are implemented in NMSSMCALC, but not yet in NMSSM-FeynHiggs. Conversely, the corrections beyond $\mathcal{O}\left(\alpha_{t} \alpha_{S}\right)$ implemented in NMSSM-FeynHiggs, which are taken over from the MSSM, have been omitted for this comparison, and their numerical impact on the SM-like Higgs-boson mass has briefly been discussed. In the final step we used the different renormalization schemes of the top/stop sector that are implemented in NMSSMCALC (but not in NMSSM-FeynHiggs). We compared the results of the OS renormalization (as obtained before) with the results using a $\overline{\mathrm{DR}}$ renormalization in the top/stop sector. Differences of $\mathcal{O}(1 \mathrm{GeV})$ have been found, are indicative of the theoretical uncertainties due to the missing higher order corrections of $\mathcal{O}\left(\alpha_{t} \alpha_{s}^{2}\right)$. The differences in the choice of $\alpha_{s}$ on the other hand (see above), lead to a somewhat larger estimate of the theoretical uncertainty due to missing higher orders. In order to make contact with [55], we also analyzed the differences between $M_{H^{ \pm}}$ 
and $A_{\lambda}$ (as used in that analysis) as $\overline{\mathrm{DR}}$ inputs, which are two possible input options in NMSSMCALC. Here only very small differences for the SM-like Higgs boson have been found.

In this paper we have identified the various sources of differences between the presented calculations within an onshell scheme for the top/stop sector and between different renormalization schemes. The analyses performed in this paper yield a better understanding of the remaining theoretical uncertainties from unknown higher-order corrections in the predictions for the Higgs-boson masses and mixingmatrix elements in the NMSSM. These results can now be used to endow the theoretical predictions for observables in the NMSSM Higgs sector with reliable estimates for the remaining uncertainties.

Acknowledgements We acknowledge useful discussions with Kathrin Walz and Dao Thi Nhung. H.R.'s work is partially supported by the Danish National Research Foundation under Grant DNRF:90. The work of S.H. is supported in part by CICYT (Grant FPA 2013-40715-P) and by the Spanish MICINN's Consolider-Ingenio 2010 Program under Grant MultiDark CSD2009-00064. The work of G.W. is supported in part by the DFG through the SFB 676 "Particles, Strings and the Early Universe" and by the European Commission through the "HiggsTools" Initial Training Network PITN-GA-2012-316704. R.G. is supported by a European Union COFUND/Durham Junior Research Fellowship under the EU Grant Number 609412.

Open Access This article is distributed under the terms of the Creative Commons Attribution 4.0 International License (http://creativecomm ons.org/licenses/by/4.0/), which permits unrestricted use, distribution, and reproduction in any medium, provided you give appropriate credit to the original author(s) and the source, provide a link to the Creative Commons license, and indicate if changes were made.

Funded by $\mathrm{SCOAP}^{3}$.

\section{Appendix}

In the following we provide summary tables including the mass values and mixing-matrix elements at the two-loop level for TP2-TP5 obtained with the "out-of-the-box" and all modified versions of NMSSMCALC and NMSSM-FeynHiggs. The mass values of the SM-like scalar are written in bold fonts, those of the singlet-like scalar in italics.

See Tables 15, 16 and 17.
Table 15 Summary table for the mass predictions for the $\mathcal{C P}$-even scalars when using the indicated versions of NMSSMCALC and NMSSM-FeynHiggs as specified in Sects. 5.1 and 5.4

Table 16 Summary table for the mass predictions for the $\mathcal{C P}$-even scalars when using the $\mathrm{OS}$ and $\overline{\mathrm{DR}}$ renormalization in the top/stop sector and either $A_{\lambda}$ as input or $M_{H^{ \pm}}$as specified in Sect. 5.6

\begin{tabular}{|c|c|c|c|c|c|c|c|c|c|}
\hline & \multirow[t]{2}{*}{$Q$} & \multicolumn{2}{|l|}{ TP2 } & \multicolumn{2}{|l|}{ TP3 } & \multicolumn{2}{|l|}{$\underline{\text { TP4 }}$} & \multicolumn{2}{|l|}{ TP5 } \\
\hline & & $M_{S}$ & $m_{t}$ & $M_{S}$ & $m_{t}$ & $M_{S}$ & $m_{t}$ & $M_{S}$ & $m_{t}$ \\
\hline \multirow[t]{4}{*}{$h_{1}$} & NC OS & 120.42 & - & 89.92 & 88.81 & 126.44 & 126.65 & 119.54 & 117.63 \\
\hline & $\mathrm{NC} \alpha_{s} \bmod$ & 114.70 & - & 89.76 & 88.83 & 125.07 & 126.71 & 117.71 & 117.71 \\
\hline & $\mathrm{N}-\mathrm{FH}$ & 114.12 & - & 89.67 & 89.36 & 126.17 & 126.29 & 118.47 & 117.95 \\
\hline & $\mathrm{N}-\mathrm{FH} \alpha\left(M_{Z}\right)$ & 114.65 & - & 89.72 & 89.31 & 125.56 & 125.74 & 118.31 & 117.77 \\
\hline \multirow[t]{4}{*}{$h_{2}$} & $\mathrm{NC} O S$ & 5951.36 & - & 126.16 & 125.80 & 143.32 & 142.73 & 124.44 & 123.51 \\
\hline & $\mathrm{NC} \alpha_{s} \bmod$ & 5951.36 & - & 123.90 & 125.87 & 142.96 & 142.74 & 122.88 & 123.60 \\
\hline & $\mathrm{N}-\mathrm{FH}$ & 5951.36 & - & 124.55 & 125.02 & 143.11 & 142.68 & 122.93 & 123.10 \\
\hline & $\mathrm{N}-\mathrm{FH} \alpha\left(M_{Z}\right)$ & 5951.36 & - & 124.26 & 124.88 & 142.98 & 142.59 & 122.81 & 123.08 \\
\hline \multirow[t]{4}{*}{$h_{3}$} & NC OS & 6370.77 & - & 652.60 & 652.70 & 467.89 & 467.35 & 627.18 & 628.72 \\
\hline & $\mathrm{N}-\mathrm{FH}$ & 6370.85 & - & 652.17 & 652.65 & 467.10 & 467.33 & 626.59 & 628.76 \\
\hline & $\mathrm{NC} \alpha_{s} \bmod$ & 6370.76 & - & 652.56 & 652.70 & 467.75 & 467.35 & 627.14 & 628.72 \\
\hline & $\mathrm{N}-\mathrm{FH} \alpha\left(M_{Z}\right)$ & 6370.90 & - & 652.29 & 652.81 & 467.43 & 467.61 & 626.73 & 628.84 \\
\hline
\end{tabular}

\begin{tabular}{|c|c|c|c|c|c|c|c|c|c|}
\hline & \multirow[t]{2}{*}{$Q$} & \multicolumn{2}{|l|}{ TP2 } & \multicolumn{2}{|l|}{ TP3 } & \multicolumn{2}{|l|}{ TP4 } & \multicolumn{2}{|l|}{ TP5 } \\
\hline & & $M_{S}$ & $m_{t}$ & $M_{S}$ & $m_{t}$ & $M_{S}$ & $m_{t}$ & $M_{S}$ & $m_{t}$ \\
\hline \multirow[t]{3}{*}{$h_{1}$} & NC OS & 120.42 & - & 89.92 & 88.81 & 126.44 & 126.65 & 119.54 & 117.63 \\
\hline & $\mathrm{NC} \overline{\mathrm{DR}}$ & 118.57 & - & 90.17 & 88.94 & 126.15 & 125.90 & 119.86 & 118.56 \\
\hline & $\mathrm{NC} A_{\lambda}$ & 118.57 & - & 90.88 & 87.78 & 126.37 & 125.76 & 120.32 & 118.65 \\
\hline \multirow[t]{3}{*}{$h_{2}$} & NC OS & 5951.36 & - & 126.16 & 125.80 & 143.32 & 142.73 & 124.44 & 123.51 \\
\hline & $\mathrm{NC} \overline{\mathrm{DR}}$ & 5951.36 & - & 124.88 & 124.86 & 142.38 & 142.16 & 123.28 & 125.20 \\
\hline & $\mathrm{NC} A_{\lambda}$ & 5951.36 & - & 124.86 & 124.68 & 142.59 & 141.28 & 123.14 & 125.26 \\
\hline \multirow[t]{3}{*}{$h_{3}$} & NC OS & 6370.77 & - & 652.60 & 652.70 & 467.89 & 467.35 & 627.18 & 628.72 \\
\hline & $\mathrm{NC} \overline{\mathrm{DR}}$ & 6371.21 & - & 652.44 & 652.65 & 467.39 & 467.03 & 626.97 & 628.75 \\
\hline & NC $A_{\lambda}$ & 6371.31 & - & 652.48 & 652.64 & 467.42 & 467.01 & 627.00 & 628.77 \\
\hline
\end{tabular}


Table 17 Summary table for the absolute values for the mixing-matrix elements of the $\mathcal{C P}$-even scalar sector when using the indicated versions of NMSSMCALC and NMSSM-FeynHiggs as specified in Sects. 5.1 and 5.4

\begin{tabular}{|c|c|c|c|c|c|c|c|c|}
\hline \multirow[t]{2}{*}{$i$} & \multirow[t]{2}{*}{$Q$} & & \multicolumn{3}{|l|}{$\underline{\mathrm{TP}} 2$} & \multicolumn{3}{|l|}{ TP3 } \\
\hline & & & $\mid \overline{U_{i 1}^{h} \mid}$ & $\left|U_{i 2}^{h}\right|$ & $\left|U_{i 3}^{h}\right|$ & $\mid \overline{U_{i 1}^{h} \mid}$ & $\left|U_{i 2}^{h}\right|$ & $\left|U_{i 3}^{h}\right|$ \\
\hline \multirow[t]{8}{*}{1} & \multirow[t]{4}{*}{$M_{S}$} & NC OS & 0.1034 & 0.9946 & 0.0004 & 0.2199 & 0.1994 & 0.9549 \\
\hline & & $\mathrm{NC} \alpha_{s} \bmod$ & 0.1034 & 0.9946 & 0.0004 & 0.2243 & 0.2140 & 0.9507 \\
\hline & & $\mathrm{N}-\mathrm{FH}$ & 0.1034 & 0.9946 & 0.0004 & 0.2134 & 0.2064 & 0.9549 \\
\hline & & $\mathrm{N}-\mathrm{FH} \alpha\left(M_{Z}\right)$ & 0.1034 & 0.9946 & 0.0004 & 0.2229 & 0.2270 & 0.9480 \\
\hline & \multirow[t]{4}{*}{$m_{t}$} & NC OS & - & - & - & 0.2236 & 0.2210 & 0.9493 \\
\hline & & $\mathrm{NC} \alpha_{s} \bmod$ & - & - & - & 0.2234 & 0.2204 & 0.9495 \\
\hline & & $\mathrm{N}-\mathrm{FH}$ & - & - & - & 0.2245 & 0.2264 & 0.9478 \\
\hline & & $\mathrm{N}-\mathrm{FH} \alpha\left(M_{Z}\right)$ & - & - & - & 0.2342 & 0.2610 & 0.9365 \\
\hline \multirow[t]{8}{*}{2} & \multirow[t]{4}{*}{$M_{S}$} & NC OS & 0.0096 & 0.0006 & 1.0000 & 0.2797 & 0.9249 & 0.2575 \\
\hline & & $\mathrm{NC} \alpha_{s} \bmod$ & 0.0096 & 0.0006 & 1.0000 & 0.2756 & 0.9218 & 0.2726 \\
\hline & & $\mathrm{N}-\mathrm{FH}$ & 0.0090 & 0.0005 & 1.0000 & 0.2820 & 0.9228 & 0.2625 \\
\hline & & $\mathrm{N}-\mathrm{FH} \alpha\left(M_{Z}\right)$ & 0.0092 & 0.0005 & 1.0000 & 0.2751 & 0.9183 & 0.2846 \\
\hline & \multirow[t]{4}{*}{$m_{t}$} & NC OS & - & - & - & 0.2659 & 0.9232 & 0.2775 \\
\hline & & $\mathrm{NC} \alpha_{s} \bmod$ & - & - & - & 0.2660 & 0.9233 & 0.2769 \\
\hline & & $\mathrm{N}-\mathrm{FH}$ & - & - & - & 0.2656 & 0.9216 & 0.2831 \\
\hline & & $\mathrm{N}-\mathrm{FH} \alpha\left(M_{Z}\right)$ & - & - & - & 0.2566 & 0.9125 & 0.3185 \\
\hline \multirow[t]{8}{*}{3} & \multirow[t]{4}{*}{$M_{S}$} & NC OS & 0.9946 & 0.1034 & 0.0096 & 0.9346 & 0.3237 & 0.1476 \\
\hline & & $\mathrm{NC} \alpha_{s} \bmod$ & 0.9946 & 0.1034 & 0.0096 & 0.9347 & 0.3232 & 0.1478 \\
\hline & & $\mathrm{N}-\mathrm{FH}$ & 0.9946 & 0.1034 & 0.0090 & 0.9354 & 0.3253 & 0.1388 \\
\hline & & $\mathrm{N}-\mathrm{FH} \alpha\left(M_{Z}\right)$ & 0.9946 & 0.1034 & 0.0092 & 0.9352 & 0.3243 & 0.1423 \\
\hline & \multirow[t]{4}{*}{$m_{t}$} & NC OS & - & - & - & 0.9377 & 0.3144 & 0.1476 \\
\hline & & $\mathrm{NC} \alpha_{s} \bmod$ & - & - & - & 0.9377 & 0.3144 & 0.1476 \\
\hline & & $\mathrm{N}-\mathrm{FH}$ & - & - & - & 0.9376 & 0.3153 & 0.1467 \\
\hline & & $\mathrm{N}-\mathrm{FH} \alpha\left(M_{Z}\right)$ & - & - & - & 0.9377 & 0.3149 & 0.1467 \\
\hline \multirow[t]{2}{*}{$i$} & \multirow[t]{2}{*}{$Q$} & & \multicolumn{3}{|l|}{ TP4 } & \multicolumn{3}{|l|}{ TP5 } \\
\hline & & & $\left|U_{i 1}^{h}\right|$ & $\left|U_{i 2}^{h}\right|$ & $\left|U_{i 3}^{h}\right|$ & $\left|U_{i 1}^{h}\right|$ & $\left|U_{i 2}^{h}\right|$ & $\left|U_{i 3}^{h}\right|$ \\
\hline \multirow[t]{8}{*}{1} & \multirow[t]{4}{*}{$M_{S}$} & NC OS & 0.4813 & 0.7432 & 0.4648 & 0.2845 & 0.3943 & 0.8738 \\
\hline & & $\mathrm{NC} \alpha_{s} \bmod$ & 0.4839 & 0.7647 & 0.4255 & 0.1417 & 0.7305 & 0.6680 \\
\hline & & $\mathrm{N}-\mathrm{FH}$ & 0.4873 & 0.8022 & 0.3448 & 0.3540 & 0.7764 & 0.5214 \\
\hline & & $\mathrm{N}-\mathrm{FH} \alpha\left(M_{Z}\right)$ & 0.4872 & 0.8045 & 0.3398 & 0.3319 & 0.6217 & 0.7094 \\
\hline & \multirow[t]{4}{*}{$m_{t}$} & NC OS & 0.4766 & 0.7886 & 0.3885 & 0.3393 & 0.6991 & 0.6294 \\
\hline & & $\mathrm{NC} \alpha_{s} \bmod$ & 0.4766 & 0.7884 & 0.3900 & 0.3382 & 0.6914 & 0.6384 \\
\hline & & $\mathrm{N}-\mathrm{FH}$ & 0.4788 & 0.8104 & 0.3377 & 0.3390 & 0.7204 & 0.6051 \\
\hline & & $\mathrm{N}-\mathrm{FH} \alpha\left(M_{Z}\right)$ & 0.4779 & 0.8321 & 0.2813 & 0.3476 & 0.7708 & 0.5339 \\
\hline \multirow[t]{8}{*}{2} & $M_{S}$ & NC OS & 0.0895 & 0.4858 & 0.8694 & 0.2224 & 0.8594 & 0.4603 \\
\hline & & $\mathrm{NC} \alpha_{s} \bmod$ & 0.0672 & 0.4524 & 0.8893 & 0.3315 & 0.6008 & 0.7274 \\
\hline & & $\mathrm{N}-\mathrm{FH}$ & 0.0334 & 0.3775 & 0.9254 & 0.0564 & 0.5387 & 0.8406 \\
\hline & & $\mathrm{N}-\mathrm{FH} \alpha\left(M_{Z}\right)$ & 0.0270 & 0.3751 & 0.9266 & 0.1379 & 0.7120 & 0.6885 \\
\hline & $m_{t}$ & NC OS & 0.0411 & 0.4215 & 0.9059 & 0.0882 & 0.6425 & 0.7612 \\
\hline & & $\mathrm{NC} \alpha_{s} \bmod$ & 0.0413 & 0.4219 & 0.9057 & 0.0922 & 0.6508 & 0.7536 \\
\hline & & $\mathrm{N}-\mathrm{FH}$ & 0.0171 & 0.3761 & 0.9264 & 0.0819 & 0.6181 & 0.7818 \\
\hline & & $\mathrm{N}-\mathrm{FH} \alpha\left(M_{Z}\right)$ & 0.0122 & 0.3266 & 0.9451 & 0.0482 & 0.5540 & 0.8311 \\
\hline 3 & $M_{S}$ & NC OS & 0.8720 & 0.4600 & 0.1673 & 0.9325 & 0.3253 & 0.1568 \\
\hline & & $\mathrm{NC} \alpha_{s} \bmod$ & 0.8726 & 0.4589 & 0.1675 & 0.9327 & 0.3245 & 0.1570 \\
\hline & & $\mathrm{N}-\mathrm{FH}$ & 0.8726 & 0.4625 & 0.1571 & 0.9335 & 0.3270 & 0.1469 \\
\hline
\end{tabular}


Table 17 continued

\begin{tabular}{|c|c|c|c|c|c|c|c|}
\hline \multirow[t]{2}{*}{$Q$} & \multirow[t]{2}{*}{$Q$} & \multicolumn{3}{|l|}{ TP4 } & \multicolumn{3}{|l|}{ TP5 } \\
\hline & & $\left|U_{i 1}^{h}\right|$ & $\left|U_{i 2}^{h}\right|$ & $\left|U_{i 3}^{h}\right|$ & $\left|U_{i 1}^{h}\right|$ & $\left|U_{i 2}^{h}\right|$ & $\left|U_{i 3}^{h}\right|$ \\
\hline \multirow{5}{*}{$m_{t}$} & $\mathrm{~N}-\mathrm{FH} \alpha\left(M_{Z}\right)$ & 0.8729 & 0.4606 & 0.1611 & 0.9332 & 0.3264 & 0.1506 \\
\hline & NC OS & 0.8782 & 0.4477 & 0.1685 & 0.9365 & 0.3137 & 0.1564 \\
\hline & $\mathrm{NC} \alpha_{s} \bmod$ & 0.8782 & 0.4477 & 0.1685 & 0.9365 & 0.3138 & 0.1564 \\
\hline & $\mathrm{N}-\mathrm{FH}$ & 0.8778 & 0.4493 & 0.1662 & 0.9372 & 0.3146 & 0.1505 \\
\hline & $\mathrm{N}-\mathrm{FH} \alpha\left(M_{Z}\right)$ & 0.8783 & 0.4482 & 0.1662 & 0.9364 & 0.3147 & 0.1554 \\
\hline
\end{tabular}

\section{References}

1. ATLAS Collaboration, CMS Collaboration, ATLAS-CONF-2015044, CMS-PAS-HIG-15-002 (2015)

2. J.F. Gunion, H.E. Haber, G.L. Kane, S. Dawson, Front. Phys. 80, $1(2000)$

3. S.P. Martin, Adv. Ser. Direct. High Energy Phys. 21, 1 (2010). arXiv:hep-ph/9709356

4. S. Dawson, arXiv:hep-ph/9712464

5. A. Djouadi, Phys. Rep. 459, 1 (2008). arXiv:hep-ph/0503173

6. P. Fayet, Nucl. Phys. B 90, 104 (1975)

7. R. Barbieri, S. Ferrara, C.A. Savoy, Phys. Lett. B 119, 343 (1982)

8. M. Dine, W. Fischler, M. Srednicki, Phys. Lett. B 104, 199 (1981)

9. H.P. Nilles, M. Srednicki, D. Wyler, Phys. Lett. B 120, 346 (1983)

10. J. Frere, D. Jones, S. Raby, Nucl. Phys. B 222, 11 (1983)

11. J. Derendinger, C.A. Savoy, Nucl. Phys. B 237, 307 (1984)

12. J.R. Ellis, J. Gunion, H.E. Haber, L. Roszkowski, F. Zwirner, Phys. Rev. D 39, 844 (1989)

13. M. Drees, Int. J. Mod. Phys. A 4, 3635 (1989)

14. U. Ellwanger, M. Rausch de Traubenberg, C.A. Savoy, Phys. Lett. B 315, 331 (1993). arXiv:hep-ph/9307322

15. U. Ellwanger, M. Rausch de Traubenberg, C.A. Savoy, Z. Phys. C 67, 665 (1995). arXiv:hep-ph/9502206

16. U. Ellwanger, M. Rausch de Traubenberg, C.A. Savoy, Nucl. Phys. B 492, 21 (1997). arXiv:hep-ph/9611251

17. T. Elliott, S. King, P. White, Phys. Lett. B 351, 213 (1995). arXiv:hep-ph/9406303

18. S. King, P. White, Phys. Rev. D 52, 4183 (1995). arXiv:hep-ph/9505326

19. F. Franke, H. Fraas, Int. J. Mod. Phys. A 12, 479 (1997). arXiv:hep-ph/9512366

20. M. Maniatis, Int. J. Mod. Phys. A 25, 3505 (2010). arXiv:0906.0777

21. U. Ellwanger, C. Hugonie, A.M. Teixeira, Phys. Rep. 496, 1 (2010). arXiv:0910.1785

22. U. Ellwanger, Phys. Lett. B 303, 271 (1993). arXiv:hep-ph/9302224

23. T. Elliott, S.F. King, P.L. White, Phys. Lett. B 305, 71 (1993). arXiv:hep-ph/9302202

24. T. Elliott, S.F. King, P.L. White, Phys. Lett. B 314, 56 (1993). arXiv:hep-ph/9305282

25. T. Elliott, S.F. King, P.L. White, Phys. Rev. D 49, 2435 (1994). arXiv:hep-ph/9308309

26. P.N. Pandita, Z. Phys. C 59, 575 (1993)

27. G. Degrassi, P. Slavich, Nucl. Phys. B 825, 119 (2010). arXiv:0907.4682

28. F. Staub, W. Porod, B. Herrmann, JHEP 10, 040 (2010). arXiv: 1007.4049

29. K. Ender, T. Graf, M. Mühlleitner, H. Rzehak, Phys. Rev. D 85, 075024 (2012). arXiv:1111.4952

30. T. Graf, R. Gröber, M. Mühlleitner, H. Rzehak, K. Walz, JHEP 10, 122 (2012). arXiv:1206.6806
31. P. Drechsel, L. Galeta, S. Heinemeyer, G. Weiglein (2016). arXiv: 1601.08100

32. M.D. Goodsell, K. Nickel, F. Staub, Phys. Rev. D 91, 035021 (2015). arXiv:1411.4665

33. M. Mühlleitner, D.T. Nhung, H. Rzehak, K. Walz, JHEP 05, 128 (2015). arXiv:1412.0918

34. G. Degrassi, S. Heinemeyer, W. Hollik, P. Slavich, G. Weiglein, Eur. Phys. J. C 28, 133 (2003). arXiv:hep-ph/0212020

35. B.C. Allanach, A. Djouadi, J.L. Kneur, W. Porod, P. Slavich, JHEP 09, 044 (2004). arXiv:hep-ph/0406166

36. S. Heinemeyer, W. Hollik, G. Weiglein, Comput. Phys. Commun. 124, 76 (2000). arXiv:hep-ph/9812320

37. S. Heinemeyer, W. Hollik, G. Weiglein, Eur. Phys. J. C 9, 343 (1999). arXiv:hep-ph/9812472

38. M. Frank et al., JHEP 0702, 047 (2007). arXiv:hep-ph/0611326

39. T. Hahn, S. Heinemeyer, W. Hollik, H. Rzehak, G. Weiglein, Comput. Phys. Commun. 180, 1426 (2009)

40. T. Hahn, S. Heinemeyer, W. Hollik, H. Rzehak, G. Weiglein, Phys. Rev. Lett. 112, 141801 (2014). arXiv:1312.4937

41. H. Bahl, W. Hollik, Eur. Phys. J. C 76, 499 (2016). arXiv:1608.01880

42. http://www.feynhiggs.de. Accessed 1 June 2017

43. P. Athron, J.-H. Park, D. Stöckinger, A. Voigt, Comput. Phys. Commun. 190, 139 (2015). arXiv: 1406.2319

44. P. Athron, J.-H. Park, T. Steudtner, D. Stöckinger, A. Voigt (2016). arXiv: 1609.00371

45. J. Baglio et al., Comput. Phys. Commun. 185, 3372 (2014). arXiv: 1312.4788

46. S.F. King, M. Muhlleitner, R. Nevzorov, K. Walz, Nucl. Phys. B 901, 526 (2015). arXiv: 1508.03255

47. U. Ellwanger, J.F. Gunion, C. Hugonie, JHEP 0502, 066 (2005). arXiv:hep-ph/0406215

48. U. Ellwanger, C. Hugonie, Comput. Phys. Commun. 175, 290 (2006). arXiv:hep-ph/0508022

49. U. Ellwanger, C. Hugonie, Comput. Phys. Commun. 177, 399 (2007). arXiv:hep-ph/0612134

50. B.C. Allanach, Comput. Phys. Commun. 143, 305 (2002). arXiv:hep-ph/0104145

51. B.C. Allanach, P. Athron, L.C. Tunstall, A. Voigt, A.G. Williams, Comput. Phys. Commun. 185, 2322 (2014). arXiv:1311.7659

52. B. Allanach, A. Bednyakov, R.R. de Austri (2014). arXiv: 1407.6130

53. W. Porod, Comput. Phys. Commun. 153, 275 (2003). arXiv:hep-ph/0301101

54. W. Porod, F. Staub, Comput. Phys. Commun. 183, 2458 (2012). arXiv: 1104.1573

55. F. Staub et al., Comput. Phys. Commun. 202, 113 (2016). arXiv: 1507.05093

56. B.C. Allanach et al., Comput. Phys. Commun. 180, 8 (2009). arXiv:0801.0045

57. P.Z. Skands et al., JHEP 07, 036 (2004). arXiv:hep-ph/0311123

58. S. Borowka, T. Hahn, S. Heinemeyer, G. Heinrich, W. Hollik, Eur. Phys. J. C 74, 2994 (2014). arXiv:1404.7074 
59. G. Degrassi, S. Di Vita, P. Slavich, Eur. Phys. J. C 75, 61 (2015). arXiv: 1410.3432

60. S. Heinemeyer, W. Hollik, H. Rzehak, G. Weiglein, Phys. Lett. B 652, 300 (2007). arXiv:0705.0746

61. G. Degrassi, P. Slavich, F. Zwirner, Nucl. Phys. B 611, 403 (2001). arXiv:hep-ph/0105096

62. K.G. Chetyrkin, J.H. Kühn, M. Steinhauser, Comput. Phys. Commun. 133, 43 (2000). arXiv:hep-ph/0004189

63. H. Baer, J. Ferrandis, K. Melnikov, X. Tata, Phys. Rev. D 66, 074007 (2002). arXiv:hep-ph/0207126

64. S.F. King, M. Mühlleitner, R. Nevzorov, Nucl. Phys. B 860, 207 (2012). arXiv:1201.2671

65. F. Staub (2008), arXiv:0806.0538

66. F. Staub, Comput. Phys. Commun. 181, 1077 (2010). arXiv:0909.2863

67. F. Staub, Comput. Phys. Commun. 182, 808 (2011). arXiv: 1002.0840
68. M. Carena et al., Nucl. Phys. B 580, 29 (2000). arXiv:hep-ph/0001002

69. J. Braathen, M.D. Goodsell, P. Slavich, JHEP 09, 045 (2016). arXiv:1606.09213

70. M. Mühlleitner, H. Rzehak, M. Spira, JHEP 04, 023 (2009). arXiv:0812.3815

71. A. Brignole, G. Degrassi, P. Slavich, F. Zwirner, Nucl. Phys. B 631, 195 (2002). arXiv:hep-ph/0112177

72. A. Brignole, G. Degrassi, P. Slavich, F. Zwirner, Nucl. Phys. B 643, 79 (2002). arXiv:hep-ph/0206101

73. A. Dedes, G. Degrassi, P. Slavich, Nucl. Phys. B 672, 144 (2003). arXiv:hep-ph/0305127

74. S. Borowka, T. Hahn, S. Heinemeyer, G. Heinrich, W. Hollik, Eur. Phys. J. C 75, 424 (2015). arXiv:1505.03133 